\title{
Modeling the relevance of immune and metabolic cues in the macrophage/fibroblast interplay during fibrosis
}

\section{Elisa Setten}

IRCCS Humanitas Research Hospital

\author{
Alessandra Castagna \\ IRCCS Humanitas Research Hospital \\ Josue Nava-Sedeno \\ National Autonomous University of Mexico \\ Jonathan Weber
}

University of Haute Alsace

Roberta Carriero

Humanitas Research Hospital

Andreas Reppas

Charité

Valery Volk

Hannover Medical School

Jessica Schmitz

Hannover Medical School

Wilfried Gwinner

Hannover Medical School https://orcid.org/0000-0003-1703-893X

Haralampos Hatzikirou

Khalifa University

Friedrich Feuerhake

Hannover Medical School https://orcid.org/0000-0002-1234-982X

Massimo Locati ( $\square$ massimo.locati@unimi.it)

IRCCS Humanitas Research Hospital

\section{Article}

Keywords: fibrosis development, transcriptomic analysis, in vitro signatures

Posted Date: August 30th, 2021

DOI: https://doi.org/10.21203/rs.3.rs-826076/v1 
License: (c) (i) This work is licensed under a Creative Commons Attribution 4.0 International License. Read Full License

Version of Record: A version of this preprint was published at Nature Communications on October 30th, 2022. See the published version at https://doi.org/10.1038/s41467-022-34241-5. 
Modeling the relevance of immune and metabolic cues in the macrophage/fibroblast interplay during fibrosis

Elisa Setten ${ }^{1,2}$, Alessandra Castagna ${ }^{1}$, Josué Manik Nava-Sedeño ${ }^{3}$, Jonathan Weber ${ }^{4}$, Roberta Carriero $^{1}$, Andreas Reppas ${ }^{5}$, Valery Volk ${ }^{6}$, Jessica Schmitz ${ }^{6}$, Wilfried Gwinner ${ }^{6,7}$, Haralampos Hatzikirou $^{8}$, Friedrich Feuerhake ${ }^{6,9}$, Massimo Locati ${ }^{1,2, *}$.

${ }^{1}$ IRCCS Humanitas Research Hospital, Rozzano, Italy; ${ }^{2}$ Department of Medical Biotechnologies and Translational Medicine, Università degli Studi di Milano, Milan, Italy; ${ }^{3}$ Department of Mathematics, National Autonomous University of Mexico, Mexico City, Mexico; ${ }^{4}$ Institute for Research in Informatics, Mathematics, Automation and Signal (IRIMAS), University of Haute Alsace, Mulhouse, France; ${ }^{5}$ Charité-Universitat Medizin Berlin, Berlin, Germany; ${ }^{6}$ Institute for Pathology, Hannover Medical School, Hannover, Germany; ${ }^{7}$ Nephrology, Hannover Medical School, Hannover, Germany; ${ }^{8}$ Mathematics Department, Khalifa University, Abu Dhabi, United Arab Emirates; ${ }^{9}$ Institute for Neuropathology, University Clinic Freiburg, Freiburg, Germany.

\section{* Corresponding author:}

Locati Massimo, MD

Humanitas Research Hospital

Via Manzoni 56; I-20089 Rozzano, Italy

Tel: +39-(0)2.8224.5116; E-mail: massimo.locati@ humanitasresearch.it

ORCID: 0000-0003-3077-590X 


\section{ABSTRACT}

Fibrosis is a progressive biological process leading to organ dysfunction in different clinical settings. As fibroblasts and macrophages are known as key cellular players for fibrosis development, we adopted an in vitro model to define the functional effects of inflammation, hypoxia, and the adaptive immune context on their functional interplay with respect to fibrosis development. Transcriptomic analysis defined the impact of each parameter, acting alone or in combination, on functional properties of both cell types, exposed individually or in a cell-cell contact. These in vitro signatures were matched with transcriptomic profiles generated on lasercaptured glomeruli and cortical tubulointerstitial area isolated from human transplanted kidneys with advanced stages of glomerulosclerosis and interstitial fibrosis/tubular atrophy, two clinically relevant conditions associated with organ failure in renal allografts. In vitro signatures were also used to instruct the development of a mathematical model predicting the relevance of each parameter in fibrosis development scenario, which indicated tolerance to inflammatory infiltrates under otherwise favorable conditions and defined an operative window in which hypoxia exerts a crucial role, supported by the degree of inflammation. Observed signatures and model-based predictions strongly suggested that irreversible fibrosis development is the result of specific combinations of metabolic and inflammatory cues, which drive distinct profibrotic paths in the glomeruli and the tubulointerstitial compartments. These findings, which found confirmation in tissue-based quantitative immune-phenotyping of transplanted kidney biopsies, indicate that the combination of in vitro and in silico modeling represents a powerful systems medicine approach to dissect fibrosis pathogenesis and develop coordinated targeted approaches. 


\section{INTRODUCTION}

Fibrosis is the final state of continuous scarring that occurs normally during healing processes but also significantly contributes reducing organ function in several chronic diseases [1]. Four core mechanisms are involved: i) early inflammatory events with involvement of various immune cells, including $\mathrm{T}$ cells and macrophages $(\mathrm{M} \varphi)$; ii) activation of fibroblasts $(\mathrm{Fb})$ to myofibroblasts (collagen-secreting $\alpha$-SMA ${ }^{+}$activated $\mathrm{Fb}$ ) and extracellular matrix (ECM) deposition, generating interstitial scars; iii) loss of epithelial cells regenerative properties; iv) loss of interstitial capillary integrity, compromising oxygen delivery and leading to a cascade of events related to hypoxia-oxidant stress, further promoting the fibrotic process [2]. Chemokines and profibrotic cytokines tightly control recruitment and activation of inflammatory cells at the site of

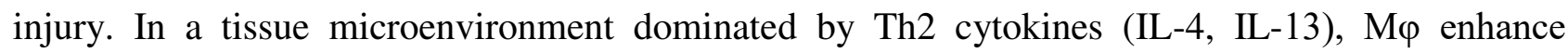
secretion and activation of latent TGF $\beta 1, \mathrm{Fb}$ proliferation and ECM production, conversion of epithelial cells into collagen-producing myofibroblasts via epithelial-mesenchymal transition, and release of proangiogenic factors to promote vascular remodeling and angiogenesis [3-5]. Pathologic angiogenesis and vessel sprouting in hypoxic tissues worsen fibrosis by leading to continuous myofibroblast activation and proliferation [6-9].

Fibrosis characterizes the progression of chronic diseases occurring in many different tissues, including skin, lung, liver, heart, and kidney [1]. In chronic kidney diseases, in particular, fibrosis can result in glomerulosclerosis with loss of glomerular filter capacity and interstitial fibrosis and tubular atrophy (IF/TA), which is associated with impaired tubular adsorption and secretion processes and regulatory functions [10]. Accumulation of ECM components in glomeruli and in the cortical interstitium collectively results in progressive loss of renal function [11]. The role of $\mathrm{M} \varphi$ in acute rejection of kidney transplant, through their contribution to both $\mathrm{T}$ cellmediated and antibody-mediated rejection processes, is well established [12,13]. On the opposite, 
their role in IF/TA development is still under debate, since positive and negative effects of $\mathrm{M} \varphi$ infiltration on long-term renal allograft functions were reported [12,14-16]. In IF/TA, M $\varphi$ have been shown to switch from a classical proinflammatory phenotype (also alluded to as M1) to an alternative phenotype (also alluded to as M2) typically associated with fibrosis progression [17,18] prompted by the release of profibrotic factors, such as TGF $\beta 1$, FGF2 and PDGF, that promote myofibroblasts proliferation and activation, leading to ECM overproduction [19]. However, in different models, chronic inflammation and subsequent fibrosis have been shown to be promoted by depletion of $\mathrm{M} \varphi$ in certain stages of the disease, and in several diseases an increase in M2 M $\varphi$ characterizes the recovery phase [20-23].

Based on the in vitro analysis of $\mathrm{Fb}$ and $\mathrm{M} \varphi$ interplay in the context of wound healing and scarring processes, a mathematical model of fibrosis has been developed to translate the in vitro observations into a potential in vivo fibrotic scenario. This model predicts three functional states: a state of healing associated with modest ECM production, and two fibrotic states associated with excessive ECM production and different cellularity where a prominent myofibroblast infiltration is associated with low or high numbers of $\mathrm{M} \varphi$, termed "hot" and "cold" fibrosis, respectively [24]. This model comprehensively describes central aspects of fibrosis and potentially predicts cell networks leading to clinically observed conditions, such as overshooting keloid scarring. Its main limitation is represented by the fact that $\mathrm{M} \varphi / \mathrm{Fb}$ interactions always occur in the context of specific immunological and metabolic cues characterizing the tissue microenvironment [25], an aspect that was not modelled. We here discuss a mathematical model describing $\mathrm{M} \varphi / \mathrm{Fb}$ interactions implemented with critical immune and metabolic microenvironmental cues, which can be applied to understand the impact of distinct tissue parameters on the development of "hot" and "cold" fibrosis processes. The model was developed based on in vitro evidence without a reference to a specific tissue, and was validated on ex vivo findings related to IF/TA progression and glomerulosclerosis in transplanted kidneys. 


\section{RESULTS}

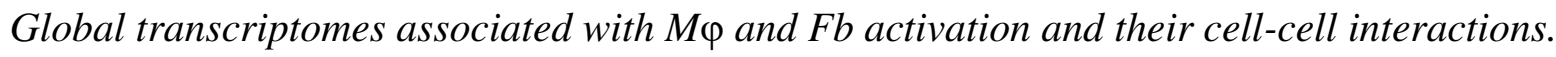

Transcriptional events related to the interaction of $\mathrm{M} \varphi$ and $\mathrm{Fb}$ in the context of defined immune and metabolic settings were investigated by RNAseq (see Fig. S1 for experimental design and sample coding). Principal component analysis (PCA) of transcriptomes associated with the 44 experimental conditions revealed that the most relevant sources of variability in the system were the cell types and their response to inflammatory conditions (PC1 and PC2, respectively, collectively accounting for $88 \%$ of total variance; Fig. 1a). Biological hallmarks were investigated by single-sample Gene Set Enrichment Analysis (ssGSEA), revealing that hypoxia, the reactive oxygen species (ROS) pathway, glycolysis, oxidative phosphorylation, fatty acid metabolism, cholesterol homeostasis, and TGF $\beta$ signaling were the major drivers of differential gene expression across of the entire dataset. In both $\mathrm{M} \varphi$ and $\mathrm{Fb}$, proinflammatory conditions were associated with IFN- and TNF-related pathways, whereas genes related to epithelial-mesenchymal transition and angiogenesis were enriched in $\mathrm{Fb}$ but not in $\mathrm{M} \varphi$ (Fig. 1b).

The contribution of the distinct parameters was then investigated by multi-level analysis to define the impact of each single variable ( $1^{\text {st }}$ level analysis) and the combinatorial effects of two $\left(2^{\text {nd }}\right.$ level analysis) or three of them ( $3^{\text {rd }}$ level analysis). The differentially expressed genes (DEGs), selected as described in Materials and methods section, were then clustered based on the main variable taken into account. When the analysis was focused on the relevance of the immune network, inflammatory/Th1conditions, mimicked by exposing cells to the combined effect of the proinflammatory mediator lipopolysaccharide (LPS) plus the type 1 cytokine IFN $\gamma$, emerged as a major driver for both cell types, generating a high number of DEGs in both $\mathrm{M} \varphi$ and $\mathrm{Fb}$. On the opposite, profibrotic/Th2conditions, mimicked by exposing cells to the type 2 cytokine IL-4, showed a divergent effect between $\mathrm{M} \varphi$, which regulated hundreds of DEGs, and Fb, which did not 
significantly differ from their untreated counterpart (Fig. 1c and 1f for $\mathrm{M} \varphi$ and Fb, respectively). The relevance of taking into consideration the combined effects of multiple parameters was exemplified by the analysis on hypoxia, whose effects in both cell types were significantly enhanced when cells were activated in coculture conditions as compared to single cell cultures (Fig. 1d and $1 \mathrm{~g}$ for $\mathrm{M} \varphi$ and $\mathrm{Fb}$, respectively). Similarly, $\mathrm{M \varphi}$ and particularly $\mathrm{Fb}$ exposed to proinflammatory conditions regulated hundreds of additional DEGs specifically when activated in the presence of the second cell type (Fig. 1e and $1 \mathrm{~h}$ for $\mathrm{M} \varphi$ and $\mathrm{Fb}$, respectively).

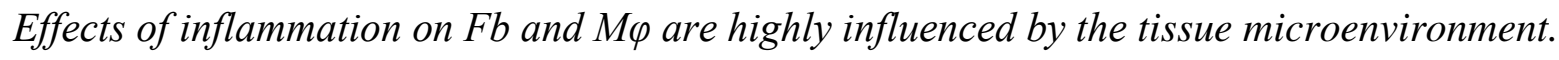

As inflammation is a potent immune driver, able to induce phenotypic and functional changes in different cell types [26,27], we first focused our analysis on its functional effects on $\mathrm{M} \varphi$ and $\mathrm{Fb}$ (Fig. 2a and 2d, respectively) comparing LPS+IFN $\gamma$-stimulated $\mathrm{M} \varphi(\mathrm{MI})$ and $\mathrm{Fb}(\mathrm{FbI})$ with resting counterparts in single culture/normoxic conditions (comparisons $A_{M}$ and $A_{F}$ ), in single culture/hypoxic conditions (comparisons $\mathrm{B}_{\mathrm{M}}$ and $\mathrm{B}_{\mathrm{F}}$ ), in coculture/normoxic conditions (comparisons $\mathrm{C}_{\mathrm{M}}$ and $\mathrm{C}_{\mathrm{F}}$ ), and in coculture/hypoxic conditions (comparisons $\mathrm{D}_{\mathrm{M}}$ and $\mathrm{D}_{\mathrm{F}}$ ). As expected, this analysis revealed that both $\mathrm{M} \varphi$ and $\mathrm{Fb}$ acquired a proinflammatory phenotype across different metabolic/culture conditions when exposed to an inflammatory setting (Fig. S2). Keeping the focus on the role of inflammation, we then applied a $2^{\text {nd }}$ level analysis comparing hypoxic versus normoxic conditions in single culture (comparison AvsB) and in coculture (comparison CvsD) conditions, and then a $3^{\text {rd }}$ level analysis comparing single culture and coculture conditions (comparison $[(\mathrm{AvsB}) \mathrm{vs}(\mathrm{CvsD})])$. This analysis revealed that a high amount of DEGs was shared among all $\mathrm{M} \varphi$ comparisons, with the remarkable exception of $\mathrm{D}_{\mathrm{M}}$, which was characterized by the emergence of 1,524 specific DEGs, revealing that even if inflammation per se is a major $\mathrm{M} \varphi$ activator the concomitant presence of $\mathrm{Fb}$ and hypoxic conditions adds further complexity to its functional effects on $\mathrm{M} \varphi$ (Fig. 2b). In line with the robustness of inflammation as $\mathrm{M} \varphi$-activating 
driver, pathway enrichment analysis revealed that in $M \varphi$ most functional pathways were linked to inflammation, adaptive immune response and cell senescence, and were shared through different experimental conditions (Fig. 2c). A remarkable exception was represented by the negative regulation in $\mathrm{M} \varphi$ of a set of functional pathways related to cholesterol metabolism when $\mathrm{M} \varphi$ were inflamed in the presence of $\mathrm{Fb}$ (comparison $\mathrm{C}_{\mathrm{M}}$ in Fig. 2c).

Similar to $\mathrm{M} \varphi$, inflammatory cues induced also in $\mathrm{Fb}$ a high number of DEGs sustaining functional pathways clearly associated with fibrosis and cell senescence (Fig. 2e and 2f). Unexpectedly, however, most of these DEGs were significantly modulated only in Fb cocultivated with $\mathrm{M} \varphi$ (comparisons $\mathrm{C}_{\mathrm{F}}$ and/or $\mathrm{D}_{\mathrm{F}}$ in Fig. 2f). Focusing on the gene enrichment analysis in $\mathrm{Fb}$ in coculture with $\mathrm{M} \varphi$, a lower activation of several pathways related to inflammation and fibrosis was evident in the concomitant presence of hypoxia (comparison $D_{F}$ in Fig. $2 f$ ), while $M \varphi$ per se had a significant impact on pathways related to cell cycle regulation and metabolism (comparison $\mathrm{C}_{\mathrm{F}}$ in Fig. 2f). These findings indicate the emergence of a senescent phenotype of both $\mathrm{M} \varphi$ and $\mathrm{Fb}$ in inflamed tissues (here mimicked by coculture conditions), with $\mathrm{M} \varphi$ modifying their metabolic profile and $\mathrm{Fb}$ regulating their cell proliferation properties, and further subtle metabolic adjustments related to the concomitant presence of hypoxia. These phenotypic changes lay the ground for assumption A of our mathematical model.

Type 2 immune responses have direct effect on $M \varphi$, which then indirectly affect $F b$.

During fibrosis development, adaptive immune responses in the site of lesion influence both immune and non-immune cells. Th2 cytokines in particular induce in $\mathrm{M} \varphi$ a phenotypic switching into an alternative phenotype (M2, here indicated as $\mathrm{MF})$, while on Fb contribute to promoting their activation into myofibroblasts involved in ECM components production (here indicated as Ff) [28]. As expected, IL-4 (here used to mimic the contribution of the Th2 immune pathway) induced a restricted but well-defined transcriptional profile in $\mathrm{M} \varphi$, which was largely insensitive to the 
hypoxic context or the concomitant presence of $\mathrm{Fb}$ (Fig. $3 \mathrm{~b}$ and comparisons $\mathrm{A}_{M}, \mathrm{~B}_{\mathrm{M}}$, and $\mathrm{C}_{M}$ in Fig. S3). On the opposite, IL-4 had no direct effect on Fb, nor was it able to induce Fb activation when hypoxia and $\mathrm{M} \varphi$ were added as single variables (Fig. $3 \mathrm{e}$ and comparisons $\mathrm{A}_{\mathrm{F}}, \mathrm{B}_{\mathrm{F}}$, and $\mathrm{C}_{\mathrm{F}}$ in Fig. S3). However, a dramatic change in the transcriptional profile of both $\mathrm{M} \varphi$ and $\mathrm{Fb}$ was observed when IL-4 was applied to both cell types under hypoxic conditions (Fig. 3b and 3e). Specifically, when cocultivated in hypoxia and in the presence of $\mathrm{Fb}$, IL-4-conditioned $\mathrm{M} \varphi$ showed a negative regulation of pathways linked to ECM deposition. A similar effect was observed in IL-4conditioned $\mathrm{Fb}$, which also showed a negative regulation of inflammation- and cell growth-related pathways specifically when exposed to hypoxia and in the concomitant presence of $\mathrm{M} \varphi$. Under these experimental conditions, both cell types also showed a significant regulation of functional pathways involved in cell-cell contacts, indicating that a direct $\mathrm{M} \varphi / \mathrm{Fb}$ interaction may be involved. Thus, at least in this in vitro setting, the development of a Th2 immune response per se has distinct effects on $\mathrm{M} \varphi$ and negligible effects on $\mathrm{Fb}$, but when hypoxic conditions are imposed to the tissue (i.e. $\mathrm{M} \varphi$ and $\mathrm{Fb}$ are allowed to directly interact with each other) IL-4 profoundly influences both inflammatory and profibrotic potential of both cell types.

Hypoxia effects are insensitive to the specific immune microenvironment but critical to cell-cell interactions.

Reduction of oxygen tension induces a metabolic switch in cells [29-31]. In our experimental setting, hypoxia $\left(1 \% \mathrm{O}_{2}\right)$ regulated a restricted set of DEGs in $\mathrm{M} \varphi$ and $\mathrm{Fb}$, including well-known hypoxia-responsive genes such as GLUT-1, VEGFA, CXCR4, and BNIP3 (Fig. S1c-e and S1f-h for $\mathrm{M} \varphi$ and $\mathrm{Fb}$, respectively). When we compared the global effect of hypoxia in single cell cultures (Fig. 4 and S4; comparisons $\mathrm{A}_{\mathrm{M}}$ and $\mathrm{A}_{\mathrm{F}}$ for $\mathrm{M} \varphi$ and $\mathrm{Fb}$, respectively) with its effects in coculture setting (Fig. 4 and $\mathrm{S} 4$; comparisons $\mathrm{B}_{\mathrm{M}}$ and $\mathrm{B}_{\mathrm{F}}$ for $\mathrm{M} \varphi$ and $\mathrm{Fb}$, respectively), we observed a dramatic increase in hypoxia-regulated DEGs when the two cell types were stimulated in tissue-like 
conditions (Fig. 4b and 4e, respectively). Conversely, the concomitant presence of proinflammatory conditions had no significant impact on the effects of hypoxia on neither $\mathrm{M} \varphi$ nor $\mathrm{Fb}$, neither in single cell nor in coculture conditions (comparisons in Fig. $S 4 ; C_{M}$ and $D_{M}$ for $M \varphi$ and $C_{F}$ and $D_{F}$ for $\mathrm{Fb}$, respectively). Similar findings were obtained when the effects of IL-4 were analyzed (data not shown).

Functional analysis performed on $\mathrm{M} \varphi$ hypoxia-responsive DEGs showed that hypoxia regulated pathways were related to actin remodeling, extracellular matrix deposition, and proliferation (Fig. 4c). Of note, these effects of hypoxia were evident only when $\mathrm{M} \varphi$ were cocultivated with $\mathrm{Fb}$, and were abolished when cells were exposed to proinflammatory or Th2 immune stimuli (Fig. 4 and S4). A similar regulation of hypoxia effects on the cell transcriptome was observed in $\mathrm{Fb}$, where hypoxia regulated DEGs related to inflammation and leukocyte recruitment (Fig. 4f).

We conclude that hypoxia has a deep impact on both $\mathrm{M} \varphi$ and $\mathrm{Fb}$ specifically when these cells can establish intercellular networks, with $\mathrm{M} \varphi$ acquiring an active and proliferative phenotype and $\mathrm{Fb}$ assuming proinflammatory and angiogenic properties. These findings constitutes the basis for assumption B of our mathematical model. Our results also suggest that this effect is mostly relevant in tissues exposed to hypoxic conditions in the absence of significant immune reactions, while the specific contribution of hypoxia to the cells transcriptome is significantly reduced when inflammatory/immune triggers concomitantly act on $\mathrm{M} \varphi$ and $\mathrm{Fb}$.

Functional interactions between $M \varphi$ and Fb are enhanced in hypoxic and inflammatory conditions. Cellular communication is a key element to adopt appropriate complex responses to stimuli of various origin (mechanic, chemical, immune, metabolic) [32]. We took advantage of our in vitro setting to specifically investigate how an immune cell, such as the M $\varphi$, interacts with a stromal cell, represented by the $\mathrm{Fb}$, in different immune and metabolic contexts and how this interaction affects 
the response to these cues. The comparison of cocultivated $\mathrm{M} \varphi$ and $\mathrm{Fb}$ to their single cultivated counterparts revealed that in resting conditions (i.e. normoxia, absence of inflammation and immune challenging) cocultivated cells did not differ from single cultivated counterparts $\left(1^{\text {st }}\right.$ level analysis; comparisons $\mathrm{A}_{\mathrm{M}}$ and $\mathrm{A}_{\mathrm{F}}$ in Fig. 5 and Fig. S5). Coculture also did not affect $\mathrm{M} \varphi$ response to inflammatory triggers, while the presence of $\mathrm{M} \varphi$ has significant impact on $\mathrm{Fb}$ response to inflammation (comparisons $\mathrm{C}_{\mathrm{M}}$ and $\mathrm{C}_{\mathrm{F}}$ in Fig. 5, respectively, and Fig. S5). At variance, coculture significantly affected the activation of both cell types in response to hypoxia, both in the absence (comparisons $\mathrm{B}_{\mathrm{M}}$ and $\mathrm{B}_{\mathrm{F}}$ in Fig. 5 and $\mathrm{S} 5$ ) and in the concomitant presence of hypoxia and inflammation (comparisons $\mathrm{D}_{\mathrm{M}}$ and $\mathrm{D}_{\mathrm{F}}$ in Fig. 5 and S5). Of note, coculture in Th2-conditioned environment induced no changes nor in $\mathrm{M} \varphi$ neither in $\mathrm{Fb}$; however, IL-4-conditioned $\mathrm{M} \varphi$ in hypoxic and tissue-like environment are able to block the switch of $\mathrm{Fb}$ into proinflammatory phenotype. We conclude that $\mathrm{Fb}$ have an impact on $\mathrm{M} \varphi$ activation only if this interplay takes place in hypoxic conditions, while $\mathrm{M} \varphi$ influence $\mathrm{Fb}$ response to hypoxia and to inflammation independently from oxygen tension levels.

Functional analysis showed that, when cells were challenged with hypoxia, coculture significantly enriched the expression of genes related to intercellular communication in both $\mathrm{M} \varphi$ and $\mathrm{Fb}$ (Fig. 5c and 5f). These functional networks related to cell-cell interactions are likely responsible for the enhanced expression of genes related to cell proliferation observed in $\mathrm{M} \varphi$ exposed to hypoxia in the presence of $\mathrm{Fb}$ and genes related to inflammation, particularly evident in $\mathrm{Fb}$ exposed to hypoxia in the presence of $\mathrm{M} \varphi$. When hypoxia operates in an inflammatory setting, a reduction in the number of enriched pathways was observed both in $\mathrm{M} \varphi$ and $\mathrm{Fb}$. In functional terms, whereas in these experimental conditions cocultivated $\mathrm{Fb}$ maintained an enriched expression of genes related to inflammation, cocultivated $M \varphi$ showed a significant enrichment only in pathways related to cell-cell interaction and hypoxia, while a gene signature related to cell proliferation was not evident anymore. Finally, $\mathrm{Fb}$ were susceptible to signals derived from the 
presence of $\mathrm{M} \varphi$ also in inflammatory settings, irrespective of the presence of hypoxia. In this setting, their response showed an upregulation of genes mainly related to cell cycle control, cell response to stress, and senescence. We conclude that in normoxic conditions the establishment of an inflammatory milieu in the tissue supports the ability of $\mathrm{M} \varphi$ to induce cellular stress and activate senescence programs in $\mathrm{Fb}$. On the opposite, in absence of an immune challenge, the interplay between $\mathrm{Fb}$ and $\mathrm{M} \varphi$ occurring in tissues in response to hypoxia modifies the response of both cell types, promoting the acquisition of proliferative properties in $\mathrm{M} \varphi$ and inducing a proinflammatory phenotype in $\mathrm{Fb}$. Of note, these effects of hypoxia are significantly attenuated when the $\mathrm{M} \varphi / \mathrm{Fb}$ interplay occurs in an inflammatory setting. These changes in cell response will be applied as assumptions $\mathrm{B}$ and $\mathrm{C}$ in the mathematical model.

Different tissue districts in fibrotic kidneys are characterized by distinct patterns of immune infiltrate and transcriptional profiles.

These in vitro results indicate the interaction between $\mathrm{Fb}$ and $\mathrm{M} \varphi$ profoundly influence the functional effects of immunological and metabolic cues operating in tissues, likely affecting their role in fibrosis development. Therefore, translation of these in vitro findings to specific human diseases requires considering the fine anatomy of the involved organ, which influences the likelihood of direct $\mathrm{M} \varphi / \mathrm{Fb}$ interactions and the degree of hypoxia.

The kidney is composed of different tissue compartments, which in the cortical area include the glomeruli and the tubulointerstitial compartments. It also presents a unique type of vascularization, with each individual glomerulus connected to the circulation by an exclusive afferent and efferent arteriolar vessels, the latter continuing into longitudinally arranged capillaries along the tubules. The distinct anatomy of the interstitial compartment and the presence of kidneyspecific epithelial boundary structures (e.g., the Bowman capsule confining glomeruli) were our rationale to investigate the potential relevance of tissue organization for fibrosis development. We 
therefore investigated the cellular composition of different compartments of the cortical tissue by multiplexed immunohistochemistry (mIHC) and used laser capture microdissection (LCM) to enrich transcriptional programs in distinct anatomic districts defined as reported in the Materials and methods section: the glomerulus; the 200-250 $\mu \mathrm{m}$ surrounding area (from now referred to as "surrounding"), which represent the range where cytokine/chemokine gradients control motility of immune cells and their mutual communication [33,34]; the tubulointerstitial area (from now referred to as "interstitium"). Comprehensive immune cell phenotyping and transcriptional analysis was performed in these anatomical areas on transplanted kidneys at different stages of fibrosis and in control samples, represented by non-fibrotic tissue specimen obtained from tumor-distant areas of kidneys removed for renal cell carcinomas.

Conventional microscopy and mIHC showed that different stages and degrees of fibrosis coexist within a specimen. Some morphologically almost intact glomeruli and normal-appearing tubular structures were still present even in kidneys with terminal graft failure after different types of chronic rejection (Fig. 6a/b). In an earlier stage of fibrosis, a broad range from severely affected to almost intact glomeruli (Fig. 6c, right upper panels) and locally different degrees of inflammation in interstitial areas (Fig. 6c, right lower panels) were present. Spatial heterogeneity between tissue compartments was also observed for the composition of the immune cell infiltrate (Fig. 6d). CD4 ${ }^{+}$ and $\mathrm{CD}^{+} \mathrm{T}$ cells showed increased density in the interstitium compared to the glomerular compartment, and $\mathrm{CD} 20^{+} \mathrm{B}$ cells were almost exclusively present outside the glomeruli. $\mathrm{M} \varphi$ subsets also showed tissue compartment-specific distribution, with abundant $\mathrm{CD} 206^{+} / \mathrm{CD} 8^{+}$alternatively activated $\mathrm{M} \varphi$ highly represented in the interstitium and almost absent in the glomeruli (Fig. 6d). In contrast, activated $\left(\mathrm{FAP}^{+}\right) \mathrm{Fb}$ were present in all compartments, but significantly enriched in the glomeruli (Fig. 6g). Large-scale neighborhood analysis revealed that: i) CD20+ B cells were largely clustered with themselves, with few $\mathrm{M} \varphi$ in the direct neighborhood; ii) mixed infiltrates composed of $\mathrm{CD}^{+}$effector and $\mathrm{CD} 4^{+}$helper $\mathrm{T}$ cells tended to cluster together with dispersed $\mathrm{M} \varphi$ of different 
types; iii) $\mathrm{FAP}^{+} \mathrm{Fb}$ were in relatively close contact to other immune cells in the interstitial area, while being predominantly located in neighborhood with each other in glomeruli, suggesting that their activation mechanisms were different in distinct tissue compartments (Fig. 6h-j).

As different tissue compartments showed different patterns of immune infiltrate, we compared the LCM-enriched sub-compartment-specific transcriptome profiles of the three distinct anatomical regions of interest (Fig. S6a). PCA showed that the anatomical area was the first source of variation among all samples, and that for each anatomical district investigated samples from control and fibrotic kidneys were clearly distinct (Fig. S6b). Gene ontology (GO) and pathways enrichment analysis revealed a number of functional categories significantly enriched in fibrotic samples, most of which related to immune response and ECM organization (Fig. S6c). We then investigated the potential relevance in vivo of the different cellular networks identified in vitro by defining their relative contribution to the transcriptional profiles detected in fibrotic reactions occurring in these different anatomical districts. To this purpose, GSEA and overlap analysis were applied to define the enrichment of specific signatures identified by in vitro comparisons in the ex vivo signature defined by the comparison between fibrotic kidneys and controls. Overall, our in vitro signatures were able to explain $45.3 \%$ of total DEGs that discriminated fibrotic kidneys from controls. This percentage included genes shared by more than one signature (35.6\%) as well as genes only present in a single signature (9.7\% in total). A limited number of experimental in vitro conditions could explain each a significant fraction (up to 20\%) of the ex vivo gene signature (Fig. S6d, middle panel). Most of these gene sets were shared among two or more settings, but some provided a univocal contribution (i.e. DEGs detected in the ex vivo signature only present in one specific in vitro experimental comparison) shading light on the biological processes occurred in the tissue. Gene sets with a univocal signature were grouped depending on the discriminating variable that defines in vitro comparison. We observed that univocal signatures that better describe fibrotic kidneys presented inflammation as a major trigger, being other variables investigated in the in vitro 
system (IL-4, cell-cell interaction, hypoxia) able to explain only a minor number of genes characterizing fibrotic kidneys (Fig. S6d, left panel). Considering both univocal and general contribution of in vitro signatures, we observed that genes associated with leukocyte activation and inflammatory events occurring in fibrotic kidneys were mostly overlapping with our in vitro data related to $\mathrm{M} \varphi$ subjected to inflammatory stimuli mainly in coculture setting, independently of oxygen level. On the other side, in vitro signatures describing $\mathrm{Fb}$ subjected to $\mathrm{M} \varphi$ influence in hypoxic environment provided a description of fibrotic and angiogenic properties characterizing fibrotic kidneys compared to control samples (Fig. S6d, left and middle panels).

\section{Enrichment of proinflammatory signatures outside glomeruli during renal allograft rejection.}

When distinct anatomical regions were investigated, PCA confirmed the clustering of fibrotic and control samples in each district (Fig. 7a, 8a and 9a for the interstitium, the surrounding area, and glomeruli, respectively). Both in the interstitium and in surrounding regions we confirmed the enrichment of inflammatory pathways in pathological samples, with GO terms related to leukocyte activation and migration, adaptive immune response, cellular response to IFN $\gamma$, regulation of cytokine production being clearly enriched in fibrotic samples (Fig. $7 \mathrm{~b}$ and $8 \mathrm{~b}$ ). Our in vitro signatures explained about $60 \%$ of the fibrosis-related signatures emerging in these two anatomical districts, with an evident predominance of signatures related to inflammation testified by $10 \%$ of DEGs included in functional categories emerging exclusively in response to inflammatory triggers used in vitro. In both anatomical districts, the in vitro generated signature contributing the most to DEGs observed in vivo was related to $\mathrm{M} \varphi$ response to inflammatory stimuli, requiring however the concomitant presence of $\mathrm{Fb}$ and hypoxia (MIvsM0_CC/24H signature; Fig. 7c and 8c). Of note, in the interstitium all signatures providing significant contribution to explain DEGs observed ex vivo were related to response to inflammation, either by $\mathrm{M} \varphi$ or $\mathrm{Fb}$. At variance, a distinct signature associated with $\mathrm{M} \varphi$ response to IL-4, again requiring the concomitant presence of $\mathrm{Fb}$ and hypoxia, 
emerged only in the surrounding tissue (MFvsM0_CC/24H signature; Fig. 8c, left and middle panels), and appeared to be specifically related to the GO category ECM organization (Fig. 8c, right panel). We conclude that, both in the interstitium and in the surrounding area, proinflammatory signatures explain most of DEGs characterizing fibrotic samples, with many DEGs provided by inflammatory $\mathrm{M} \varphi$ activated in the presence of hypoxia (Fig. 7c and 8c, right panels). Interestingly, the presence only in the surrounding area of a signature associated with $\mathrm{M} \varphi$ response to IL-4 suggests that different biological networks support fibrosis development in these two anatomical districts.

Enrichment of IL-4 conditioned signature in fibrotic glomeruli during renal allograft rejection.

Pathological glomeruli from fibrotic kidneys were clearly distinct from the corresponding control samples (Fig. 9a) and showed enrichment in pathways related to ECM organization, blood vessel morphogenesis, and cellular adhesion (Fig. 8b). In vitro signatures were clearly different from signatures that enriched the other renal districts previously described. Indeed, the contribution of inflammation-related pathways was reduced (1.2\%; Fig. 9c, left panel), and a unique signature corresponding to IL-4-activated $\mathrm{M} \varphi$ in the concomitant presence of $\mathrm{Fb}$ and hypoxia emerged, which contributed the most to describe DEGs enriched in the glomeruli area from fibrotic kidneys (MFvsM0_CC/24H; Fig. 9c, left and central panels). Of note, this signature explained more than $50 \%$ of DEGs that contributed to GO terms related to ECM deposition and blood vessels in these pathological samples (Fig. 9c, right panel). Signatures related to $\mathrm{Fb}$ interacting with $\mathrm{M} \varphi$, in hypoxic conditions but independently from inflammation, also significantly contributed to the description of glomeruli from fibrotic kidneys (CCvsSC_FbI/24H, 24Hvs24N_Fb0/CC, CCvsSC_Fb0/24H; Fig. 9c, left and central panels). Interestingly, these Fb-related in vitro signatures explained a large fraction (from $30 \%$ to $70 \%$ ) of DEGs associated with different GO categories related to cytokine signaling, immune response, cell adhesion, and chemotaxis (Fig. 9c, right panel). However, while 
all Fb-related GO categories were not specific for pathological glomeruli, as genes that enriched those pathways were also contributing to the interstitium and surrounding areas, most $\mathrm{M} \varphi$-related signatures were only evident in this anatomical compartment, suggesting that IL-4 conditioned M $\varphi$ interacting with $\mathrm{Fb}$ in hypoxic areas provide a specific spatial-restricted driver in the glomeruli area in fibrotic kidneys.

A mathematical model based on in vitro signatures identifies three dynamic states in fibrosis development.

Having defined in our in vitro model the key parameters driving the interplay between $\mathrm{M} \varphi$ and $\mathrm{Fb}$ in different biological settings, their specific functional implications, and their correspondence with fibrotic events occurring in vivo in different anatomical regions, we wondered whether this information could be applied to dissect dynamics of fibrosis development. To this scope, we developed a mathematical model able to translate the $\mathrm{M} \varphi / \mathrm{Fb}$ interactions identified in vitro into their corresponding in vivo dynamics (Fig. 10a; details are provided in Materials and methods section). One should note that the model parameters have not been calibrated to in vitro kinetics but the parametric space was exhaustively explored. Specifically, the model was based on literature information showing that inflammatory $\mathrm{M} \varphi$ are mainly sustained by local recruitment in inflamed tissues while alternative $\mathrm{M} \varphi$ accumulation can be sustained by their proliferative abilities [35], and that $\mathrm{M} \varphi$ phenotypic switching can occur relatively fast and at shorter time point compared to their proliferation/death rate [36], integrated by the three key observations emerged in our in vitro analysis related to inflammation and hypoxia:

A. when inflammatory conditions develop in the absence of significant hypoxia, both $\mathrm{M} \varphi$ and $\mathrm{Fb}$ develop a proinflammatory phenotype but also activate a senescence program (comparisons $\mathrm{C}_{\mathrm{M}}$ and $\mathrm{C}_{\mathrm{F}}$ in Fig. 2c and 2f); 
B. in hypoxic conditions, $\mathrm{M} \varphi$ acquire proliferative properties while $\mathrm{Fb}$ assume a proinflammatory phenotype (comparisons $\mathrm{B}_{\mathrm{M}}$ and $\mathrm{B}_{\mathrm{F}}$ in Fig. $4 \mathrm{~F}$ and $4 \mathrm{~L}$, comparisons $\mathrm{B}_{\mathrm{M}}$ and $\mathrm{B}_{\mathrm{F}}$ in Fig. 5c and 5f);

C. when inflammatory conditions and hypoxia are combined, $\mathrm{Fb}$ remain proinflammatory while $\mathrm{M} \varphi$ become involved in regulation of epithelial-mesenchymal transition and angiogenesis (comparisons $\mathrm{D}_{\mathrm{M}}$ and $\mathrm{D}_{\mathrm{F}}$ in Fig. 5c and 5f).

The above has been translated into the assumptions A1-A5, as shown in the Materials and methods section, which allowed us to build our mathematical model. After model analysis, we identified three dynamic phases in the inflammation/hypoxia interplay (Fig. 10b), which were reflected in renal biopsies representative of cases with different clinical courses (Fig. 10c) and in cases experiencing clinically manifest rejection (Fig. 10d).

Phase I is characterized by a low degree of hypoxia, independently of inflammation that could range from low to high levels (green area/panel in Fig. 10b and 10d, respectively). This regime is characterized by model conditions where the predictive cell trajectory starts with an arbitrary number of cells and ends with a particular amount of $\mathrm{M} \varphi$ dependent on the local inflammatory signals, while all conditions are characterized by a very low number of $\mathrm{Fb}$. A specific example of a clinical situation reflecting this stable quasi-healthy state can be found in "protocol" biopsies, which are obtained from patients at a defined time point after uncomplicated renal transplantation, as exemplified by cases of variable inflammation in the protocol biopsy six months after renal transplantation and favorable long-term clinical course (stable renal function over 2-5 years as shown in nine exemplary cases(cases 1-9, Fig. 10c): various levels of inflammation can be present, but since there is no relevant hypoxia the system can always return to normal. Another example consistent with phase I could be considered "time-point zero" biopsies taken at very early time points during or shortly after the uncomplicated surgical procedure as shown in two exemplary cases (case 10 and 11, Fig. 10d, green upper panel). In this setting, very close to the physiological 
situation of the kidney in the donor, the recipient's immune system did not yet have time to respond to the allograft, and consequently we do not yet observe severe inflammation.

Phase II, a bistable dynamic regime that can lead either to healthy or fibrotic states, is characterized by wide range of tolerable inflammation and hypoxia levels (blue area/panel in Fig. $10 \mathrm{~b}$ and $10 \mathrm{~d}$, respectively). The critical quantity for this regime is the initial condition of $\mathrm{M} \varphi$ and $\mathrm{Fb}$. In particular, there is a separatrix indicating that only high $\mathrm{M} \varphi / \mathrm{low} \mathrm{Fb}$ or vice versa may lead to a healthy renal state, with all the other initial conditions with moderate or high $\mathrm{M} \varphi$ and $\mathrm{Fb}$ levels are predicted to evolve into fibrosis development. Clinical examples reflecting such bistable condition include so-called "borderline" states, mostly observed in "indicated" biopsies that are obtained for diagnostic purposes in clinical situations requiring a decision on potential adaptation of the current immunosuppressive therapy. "Borderline" is a microscopic pattern defined by the Banff classification as evident inflammation that is suspicious, but not sufficient to justify the diagnosis of rejection. Borderline states, but also some cases of manifest rejection can still resolve, e.g. case 11 that resolved into long-term sufficient renal function after "borderline" diagnosis in an indicated biopsy after 65 months in (Fig. 10d). Both clinical conditions, borderline and some cases of manifest rejection can be successfully treated and may return to a healthy state, but a chance of turning into a profibrotic state remains.

Phase III (red area/panel in Fig. 10b and 10d, respectively) is the fully pathological, compromised state with an unfavorable combination of hypoxia and inflammation. It can be associated with "hot" or "cold" fibrosis (with or without persisting presence of immune cells, respectively). In case of "hot" fibrosis, both $\mathrm{M} \varphi$ and $\mathrm{Fb}$ are highly concentrated, whereas in case of "cold" fibrosis only the number of Fb remains high. Exemplary cases that clinically represent such states include persistent viral infection with BK virus (Fig 10d, case 10, left panel of the clinical examples) and irreversible end-stages after chronic rejection (Fig. 6a\&b). 
The mathematical model predicts a potential favorable outcome for phases I and II, which is consistent with the clinical observation of successful transplants in case of high $\mathrm{M} \varphi$ infiltration but low $\mathrm{Fb}$ number, even in the presence of dense immune cell infiltrates (Fig. 10c). Of note, we found an explicit equation separating stable (phases I and II) from clinically relevant conditions (phase III) which dictates:

$$
\text { Hypoxia }<\frac{\text { Pro }- \text { inflammatory activity }}{\text { Fibroblast viability }}
$$

Where proinflammatory activity corresponds to the combined effect of proinflammatory $\mathrm{M} \varphi$ polarization $(\lambda)$, pro-inflammatory $\mathrm{T}$ and $\mathrm{B}$ cells presence $(\mathrm{y})$ and anti-inflammatory decay rate and $\mathrm{Fb}$ viability is the ratio of $\mathrm{Fb}$ proliferation/apoptosis (mathematical details are in Materials and methods section). 


\section{DISCUSSION}

Although current treatments for fibrotic diseases typically target the inflammatory response, there is accumulating evidence that the mechanisms driving fibrogenesis are distinct from those regulating inflammation [1]. The key cellular mediator of fibrosis is the myofibroblast, which is activated by a variety of mechanisms including autocrine factors and paracrine signals derived from immune cells. In particular, in tissue repair and fibrosis $\mathrm{M} \varphi$ exert relevant effects, which go beyond their proinflammatory activities and include Fb activation [37]. As highlighted by Adler et al. [24], the reciprocal $\mathrm{M} \varphi-\mathrm{Fb}$ interaction is a key component for understanding observations such as the fibrotic time-window, the long timescale of scar maturation, and the paradoxical effect of $\mathrm{M} \varphi$ depletion. Equally important are the environmental metabolic status and the cytokines/growth factors networks, being these parameters capable to influence $M \varphi$ functions and their ability to interact with neighboring cells, including $\mathrm{Fb}$, as well as other immune cells such as lymphocytes. In our study, we implemented these immune and metabolic stimuli in a simplified in vitro system where cell circuits recreated a tissue-like scenario, giving at the same time the possibility to discriminate the weight of single variables and their combinatorial effects in the progression of the fibrotic process.

$\mathrm{M} \varphi$ and $\mathrm{Fb}$ were not influencing one to each other when interacting in a normoxic and uninflamed microenvironment, suggesting that cellular contacts without external stimuli are insufficient to induce major phenotype/functional changes. As reported, hypoxic conditions per se were able to activate a specific signature in both cell types, but surprisingly when the hypoxic environment acted on the two cell types directly interacting with each other, both $\mathrm{M} \varphi$ and $\mathrm{Fb}$ regulated a significantly larger set of DEGs. This effect constitutes the first observation for our mathematical predictive model. Similarly, the well-known strong response of $\mathrm{M} \varphi$ to inflammatory mediators was highly influenced by the presence and absence of hypoxia and $\mathrm{Fb}$. The concomitant 
exposure to inflammatory stimuli and hypoxic coculture conditions also influenced $\mathrm{Fb}$, which acquired a clear proinflammatory phenotype, while $\mathrm{M} \varphi$ remained involved in ECM remodeling and angiogenesis. This effect constitutes the second observation for the mathematical predictive model. Instead, when $\mathrm{M} \varphi$ and $\mathrm{Fb}$ were cocultivated in normoxic but proinflammatory environment, they both acquired proinflammatory properties, suggesting that in this context proinflammatory factors were sufficient to promote phenotypic changes without the contribution of metabolic switch induced by hypoxia. Moreover, both cell types downregulated oxidative phosphorylation and the EIF2 signaling pathways, indicating the activation of senescence processes that we suppose necessary to the exhaustion of inflammation. This effect constitutes the third observation included in the mathematical predictive model. Finally, while profibrotic stimulation by IL-4 promoted in $\mathrm{M} \varphi$ the alternative activation phenotype, which was not significantly influenced by neither the direct contact with $\mathrm{Fb}$ nor the presence of hypoxia, $\mathrm{Fb}$ conditioned with IL-4 did not respond in hypoxia, neither when in contact with M $\varphi$. However, IL-4 conditioned Fb differed from the resting ones, which changed their phenotype in hypoxic environment when in contact with M results suggest that IL-4 per se has no impact on $\mathrm{Fb}$, but through conditioning of $\mathrm{M} \varphi$ it induces an inhibitory mechanism preventing $\mathrm{Fb}$ from acquiring a proinflammatory phenotype. Indeed, we can suppose that though IL-4 is not sufficient to induce the acquisition of profibrotic properties in $\mathrm{Fb}$, through $\mathrm{M} \varphi$ it is able to influence $\mathrm{Fb}$ and their response to hypoxia.

Taken together, these observations obtained in an integrated tissue-like context candidate hypoxia, inflammation, and the Th2 cytokine IL-4 as key regulators of fibrosis. Consistent with this, the in vitro signatures generated in our in vitro model were able to describe up to $50 \%$ of the ex vivo signatures generated from laser microdissected fibrotic transplant nephrectomies, a surprisingly high percentage considering the variety of cell types and factors acting in the tissue that were not taken into account in the model. Moreover, we were able to distinguish different signatures, suggesting enrichment for variable functions, in three distinct anatomical regions of kidneys: 
interstitium, glomeruli surrounding area, and the compartment of glomeruli themselves. A prominent proinflammatory feature of the interstitial compartment, with immune response and leukocyte activation, was already evident from the GO analysis performed on the transcriptome of the nephrectomy ex vivo samples. In addition, the in vitro signatures of $\mathrm{M} \varphi$ cocultivated with $\mathrm{Fb}$ in hypoxic and proinflammatory conditions shared the proinflammatory features as major source of variation and were able to explain $2.7 \%$ of differentially expressed genes in this anatomical region univocally. Moreover, interstitium showed a higher density of $\mathrm{M} \varphi$, $\mathrm{T}$ and $\mathrm{B}$ lymphocytes than the other regions suggesting a massive inflammatory response in this area. In the glomeruli surrounding region, inflammation remained an important feature, similarly but at lower levels than in the interstitium, but in addition the transcriptomic profile of this compartment showed also a slightly increased relevance of processes linked to ECM remodeling. This corresponded to enrichment of transcriptomic signatures connected to M $\varphi$ IL-4 stimulation that were obtained by in vitro experiments under hypoxic conditions with $\mathrm{Fb}$ coculture. Finally, the transcriptome of the glomerular compartment showed a strikingly different composition: in these areas we found predominant signatures of $\mathrm{Fb}$ and enrichment for gene sets clearly suggesting profibrotic behavior with a reduction of proinflammatory properties. The trend towards gradually declining levels of inflammation from high in the interstitium, lower in glomeruli surrounding area, and lowest in glomeruli was paralleled by an opposite for signatures of fibrosis for which enrichment was least prominent in the interstitium, increased in the glomeruli surrounding area and was highest in the glomeruli. Corresponding to the transcriptomic profiles, IHC showed in some glomeruli aggregates of $\mathrm{FAP}^{+}$activated $\mathrm{Fb}$ in largely isolated localization, suggesting regions of "cold" fibrosis as proposed by Adler et al. [24], where no direct interaction with $\mathrm{M} \varphi$ or other immune cells is required to maintain a profibrotic state. Moreover, the same microscopic section of renal tissue could concomitantly include glomeruli with different density of $\mathrm{FAP}^{+}$cells, and different degrees of fibrosis. This spatial heterogeneity of the extent of fibrosis and profibrotic states within a kidney 
can be predicted, based on the mathematical model that we have developed on the basis of in vitro observations. Given that the model predicts the existence of three dynamic regimens that can modify the interplay between $\mathrm{M} \varphi$ and $\mathrm{Fb}$ depending on local hypoxia and inflammation, it can be expected that the regional outcome in a kidney with its complex vascular system, potential preexisting pathological conditions and variable presence of $\mathrm{M} \varphi$ and $\mathrm{Fb}$ due to locally different of focal inflammatory infiltrates will be heterogeneous. The predicted distinct cellular trajectories, which could lead to different degrees of stability ranging from stable healthy to irreversible pathological conditions, is reflected by immunohistological data confirming spatial heterogeneity with the coexistence of hot and cold fibrosis regions. Though based on a single case, the analysis of renal biopsies from a SV40-positive case with reactivation of the polyomavirus BK suggests that the mathematical prediction is not necessarily specific for graft rejection.

Taken together, the findings confirm that $\mathrm{Fb}$ and $\mathrm{M} \varphi$ are central to the development of fibrosis, and show that additional local microenvironmental cues such as inflammation, $\mathrm{M} \varphi$ polarization, and hypoxia have relevant impact on the regional outcome of profibrotic constellations. Consequently, therapeutic interventions in order to avoid or reduce fibrosis may have variable effects on the delicate balance between different factors, including oxygenation, the degree and type of inflammation, and local anatomical conditions in different kidney compartments. This may have some intriguing therapeutic implications. On a first note, our results confirm the plausibility of anti-inflammatory drug usage, which is the major strategy in the current Standard of Care. However, the model-based predictions reveal that a critical inflammation intensity highly depends on local hypoxia and cell interactions, which may explain why anti-inflammatory treatments may fail to fully prevent fibrosis. Moreover, interventions controlling Fb proliferation, for example PDGFR-targeted drugs such as Nintedanib, may be helpful against fibrosis development. However, the inhibitory effect on $\mathrm{Fb}$ proliferation rates and the overall outcome may again depend on local inflammation and hypoxia, implying that multimodal approaches optimizing 
immunological and metabolic cues may be the most promising application of such drugs. The same holds true for drugs modifying $\mathrm{M} \varphi$ reprogramming, which could potentially contribute to effective treatment strategies, but only under certain microenvironmental circumstances. For example, the present model would suggest that this could only be successful when $\mathrm{M} \varphi$ phenotypic switch rate $(\lambda)$ is larger than the $\mathrm{Fb}$ viability rate $\beta / \delta 2$ (proliferation/death). In conclusion, therapeutic interventions avoiding or significantly reducing development of fibrosis in anatomically complex organs like the kidney are unlikely to be successful in the setting of unimodal treatment. In vitro and in silico modelling are powerful tools to support the discovery and development of new strategies to fight fibrosis in coordinated combinations of targeted approaches. 


\section{MATERIALS AND METHODS}

\section{Cell cultures.}

Human monocytes were obtained from healthy blood donor buffy coats, upon approval by the local ethical committee. Monocytes were isolated by two-step density gradient centrifugations using Lympholyte $\mathrm{H}$ (Cederlane) and 46\%Percoll (Lonza) followed by incubation of purified cells in RPMI 1640 (Lonza) without serum, for $20 \mathrm{~min}$ at RT. Adherent monocytes were washed twice with PBS and then cultured in RPMI medium supplemented with $10 \%$ fetal bovine serum (FBS; Lonza), $100 \mathrm{U} / \mathrm{mL}$ penicillin/streptomycin (Lonza), and $2 \mathrm{mM} \mathrm{L-glutamine} \mathrm{(Lonza).} \mathrm{Monocyte} \mathrm{purity} \mathrm{was}$ $>90 \%$ as assessed by CD14/CD16 FACS analysis [38]. M $\varphi$ were obtained by culturing monocytes for 7 days in complete RPMI supplemented with human M-CSF (100ng/ml; Miltenyi). The human dermal BJ fibroblast cell line (CRL-2522; ATCC) was cultivated in high glucose D-MEM (Lonza) $10 \%$ FBS, $100 \mathrm{U} / \mathrm{mL}$ penicillin/streptomycin, and $2 \mathrm{mM} \mathrm{L-glutamine.} \mathrm{When} \mathrm{cultivated} \mathrm{in} \mathrm{normoxic}$ conditions, $\mathrm{M} \varphi$ and $\mathrm{Fb}$ were maintained at $37^{\circ} \mathrm{C}$ in a humidified incubator settled at $20 \% \mathrm{O}_{2}, 5 \%$ $\mathrm{CO}_{2}$ in air, while hypoxic treatment was performed moving cells at $37^{\circ} \mathrm{C}$ in a humidified incubator with a mixture of $1 \% \mathrm{O}_{2}, 5 \% \mathrm{CO}_{2}$ and $94 \% \mathrm{~N}_{2}$. Hypoxic and normoxic cells are labelled "H" and "N", respectively. M $\varphi$ were polarized toward a proinflammatory phenotype (MI) by incubation with $100 \mathrm{ng} / \mathrm{ml}$ LPS (Sigma) plus $20 \mathrm{ng} / \mathrm{ml}$ IFN $\gamma$ (R\&D Systems) or into an alternative phenotype (MF) by incubation with $20 \mathrm{ng} / \mathrm{ml} \mathrm{IL-4} \mathrm{(Miltenyi)} \mathrm{[39].} \mathrm{Resting} \mathrm{M \varphi} \mathrm{(M0)} \mathrm{were} \mathrm{left} \mathrm{unstimulated} \mathrm{for} \mathrm{the}$ same period. $\mathrm{Fb}$ were stimulated as $\mathrm{M} \varphi$, with cells treated with LPS+IFN $\gamma(\mathrm{FbI})$, IL-4 (FbF) or left unstimulated $(\mathrm{Fb} 0)$. Polarizing stimuli and hypoxia were applied simultaneously. For coculture experiments, differentiated $\mathrm{M} \varphi$ were replated directly onto adherent $\mathrm{Fb}$ (plated $16 \mathrm{~h}$ before) with a 2:1 ratio. After $24 \mathrm{~h}$ of coculture in basal conditions (normoxia without stimuli), cells were stimulated as described above, detached, and FACS sorted based on staining with anti-human CD45 (BD Bioscience) to distinguish $\mathrm{CD}^{4} 5^{+} \mathrm{M} \varphi$ from $\mathrm{CD}^{-} 5^{-} \mathrm{Fb}$ using a FACS Aria III cell sorter (BD 
Bioscience). Zombie Aqua Fixable Viability kit (BioLegend) was used to exclude dead cells (L/D ${ }^{+}$. Single cell and FACS-sorted cocultivated cells are labeled "SC" and "CC", respectively. The combination of different parameters applied to $\mathrm{M} \varphi$ and $\mathrm{Fb}$ generated a total of 44 different experimental conditions, detailed in Fig. S1A. The experiment was performed in triplicate and the transcriptomic profiles were then investigated.

\section{Real-time quantitative PCR ( $q P C R)$.}

Total mRNA was extracted from human $\mathrm{M} \varphi$ and fibroblasts using DirectZOL RNA miniprep kit (Zymo Research) according to the manufacturer's instructions. Reverse transcription was done using High-Capacity cDNA Reverse Transcription kit (Applied Biosystems). qPCR was performed using TaqMan Fast Advanced Master Mix 2X (Applied Biosystems) and TaqMan probes (Thermo Fisher) for Glut1 (Hs00892681_m1), Vegfa (Hs00900055_m1), and Cxcr4 (Hs00607978_s1). Reactions were performed on a VIIA-7 qPCR Detection System (Applied Biosystems). The thermal cycling conditions were standard fast-cycling. Relative expression values were calculated using the $\Delta \Delta$ CT method normalized on the housekeeping gene Gapdh (Hs_99999905_m1).

\section{Histological samples.}

Immune cell phenotyping and transcriptional analysis were performed on formalin-fixed paraffinembedded $3 \mu \mathrm{m}$ thick consecutive sections from archival material of four transplant nephrectomy specimens at different stage of fibrosis (three terminal graft kidneys with advanced fibrosis, showing TCMR, ABMR, and mixed TCMR/ABMR phenotype; one kidney with ABMR and less advanced fibrosis, surgically removed because of the development of renal cell carcinoma within the transplanted organ [40]). As non-fibrotic control samples we used tissue selected as distant as possible from the tumor margin form four tumor nephrectomy specimens. Protocol biopsies from kidney transplants were obtained from a previous study [41], and the clinical follow-up 
(development of GFR over time) was obtained in the context of the SYSIMIT systems medicine study (www.sysim.it). The study was approved by the local institutional review board (IRB; approval numbers \#2063-2013 and \#2968_2015), in alignment with the respective IRBs in the European research institutions participating in the ERACoSysMed project. Sections were stored at $4^{\circ} \mathrm{C}$ in the dark to minimize antigen aging over time until further processing.

\section{Immunohistochemistry(IHC).}

Standard histology (H\&E, PAS, sirius red) and IHC to detect (fibroblast-activating protein FAP, CD206) as well as control experiments to calibrate the multiplexing IHC for CD4, CD8, CD20, CD68, CD206, MS4A4A and FAP) were performed using an automated staining instrument (Ventana Benchmark Ultra) following the manufacturer's recommendations, and using 3,3 diaminobenzidine or Fast-Red as chromogens. Multiplexed IHC analysis was optimized following the manufacturer's instructions (OPAL Multiplex IHC Assay Development Guide, Akoya Bioscience). Slides were deparaffinized, initial antigen retrieval was performed by microwave cooking in Tris-buffered saline (TBS) at $\mathrm{pH}$ 9, and blocking of unspecific protein binding was performed using Protein Block Serum-free solution (Agilent/Dako). Subsequent antigen retrieval and deactivation of the preceding staining step was performed either in TBS at $\mathrm{pH} 9$ or citrate buffer at $\mathrm{pH}$ 6. Consecutive IHC staining using the OPAL 7-plex fluorescence system was performed using the following primary antibodies: anti-CD4 (clone SP35; Zytomed Systems), antiCD8 (clone C8/144B; Agilent/Dako), anti-CD20 (clone L26; Dako), anti-CD68 (clone PG-M1; Dako), anti-CD206 (clone 5C11; Bio-Rad), anti-FAP (clone D8; Vitatex). Cell nuclei were stained with DAPI. The following fluorophores were used in the tyramide signal amplification-based multiplexed system to detect bound antibodies: Opal 520, Opal 540, Opal 570, Opal 620, Opal 650 or Opal 690. Fluoromount-G mounting medium (Thermo Fisher Scientific) was applied to cover slides before imaging. 
Multispectral image analysis and quantitative evaluation.

Whole slide image scanning was done at 20x magnification using the Vectra Polaris instrument (Akoya Bioscience). Three-channel fluorescent whole slide images were used to select regions of interest in nephrectomies ( 466 × $349 \mu \mathrm{m}$ size) for subsequent targeted scanning of image stacks at 40x magnification across the visible spectrum $(420-720 \mathrm{~nm})$ for multispectral imaging, containing glomeruli and the surrounding region, or representative areas of the interstitial compartment. Biopsies were fully scanned and spectral libraries were generated using single stained scans of tonsil tissue for each reagent. Deconvolution and training for the machine learning-based segmentation, tissue classification and cell phenotyping algorithms were performed using the inForm v2.4.8 software (Akoya Bioscience). Visual quality control of results was performed by comparing all composite images and selected single-channel images and phenotyping results with corresponding chromogenic single and duplex staining of adjacent consecutive sections.

\section{Cell density and neighborhood analysis.}

Cell phenotypes and individual coordinates of each cell were exported in text (.csv) format and further processed in GraphPad and MS Excel. Statistical analysis was performed using GraphPad Prism version 7. Accepted significance levels were $* \mathrm{p}<0.05$, ** $\mathrm{p}<0.01$.In the context of neighborhood analysis the term "glomerulus" refers to the area within the Bowman membrane. For this analysis, the term "surrounding" refers to the area within a ROI (see above) of $466 \times 349 \mu \mathrm{m}$ size, where the glomerulus is in the center (see Fig 6a-c, panels labeled with "Glo"), subtracting the glomerulus area from each ROI. The term "interstitium" refers to ROIs of the same 466 x $349 \mu \mathrm{m}$ size that are taken exclusively in interstitial areas, strictly avoiding areas meeting the "glomerulus" or "surrounding" criteria. 
Laser-captured microdissection (LCM) and RNA sequencing.

Formalin-fixed paraffin-embedded $5 \mu \mathrm{m}$ thick tissue sections adjacent to the sectioning levels used for multiplexed IHC were mounted on a poly-1-lysin-coated membrane attached to a metal frame under RNAse-free conditions. LCM was performed on deparaffinized, hemalaun-stained sections using the Cell Cut Plus System (MMI Molecular Machines \& Industries). In the context of LCM analysis, the term "glomerulus" refers to the area within the Bowman capsule; the term "surrounding" refers to a circle with a diameter of $550 \mu \mathrm{m}$ with the glomerulus in the center, obtained after the glomerulus had been removed, and considering that in paraffin sections glomeruli show a diameter of $\sim 200 \mu \mathrm{m}$ (range $150-250 \mu \mathrm{m}$ ) and some tissue loss caused by the laser beam, in practical terms this area corresponds to a ring area of 200-250 $\mu \mathrm{m}$ around each glomerulus adopted for the cell density and neighborhood analysis; the term "interstitial" refers to areas of variable shape selected by strictly avoiding the other two compartments (Fig. S6). We chose this pragmatic terminology to make the LMD and multispectral image analysis results comparable using similar distances/areas. RNA isolation was performed using the RNeasy Mini Kit (Qiagen) in combination with Qiashredder columns (Qiagen) according to the manufacturer's instructions. Obtained RNA quality was checked using the RNA Nano Kit (Agilent Technologies) on an Agilent BioAnalyzer 2100 (Agilent Technologies). Prior to library generation, nucleic acid samples were pretreated with DNase (Qiagen). 5ng equivalents from DNase pretreated samples were used for library preparation with the SMARTer Stranded Total RNA-Seq Kit - Pico Input Mammalian (\#635006; Takara/Clontech) according to conditions recommended in user manual \#101215. Generated libraries were barcoded by dual indexing approach and were finally amplified with 15 cycles of PCR. Fragment length distribution of generated libraries was monitored using a BioAnalyzer High Sensitivity DNA assay (5067-4626; Agilent Technologies). Quantification of libraries was performed by use of the Qubit ${ }^{\circledR}$ dsDNA HS Assay Kit (Q32854; Thermo Fisher Scientific). Pooled libraries were denatured with $\mathrm{NaOH}$ and finally diluted to $1.8 \mathrm{pM}$ according to the Denature and 
Dilute Libraries Guide (document \# 15048776 v02; Illumina). $1.3 \mathrm{ml}$ of denatured pool was loaded on an Illumina NextSeq 550 sequencer using a High Output Flowcell for 75bp single reads (\#FC404-2005; Illumina). BCL files were converted to FASTQ files using bcl2fastq Conversion Software version v2.20.0.422 (Illumina). Raw data processing and quality control were conducted by use of nfcore/rnaseq (version $1.5 \mathrm{dev}$ ). The genome reference and annotation data were taken from GENCODE.org (Homo sapiens; GRCh38.p12; release 28). Normalization and differential expression analyses were performed with DESeq2 (Galaxy Tool Version 2.11.40.2) with default settings except for "Output normalized counts table" which was set to "Yes". FACS sorted samples were collected and cells were lysed by TRIzol reagent. Total RNA was isolated using DirectZOL RNA miniprep kit (ZymoResearch) according to the manufacturer's instructions. Quantification and quality check (RNA integrity number RIN > 7) were assessed by using Qubit4 (Invitrogen) instrument. Libraries preparation and processing were performed with Lexogen protocol, using the QuantSeq 3' mRNA-Seq Library Prep kit to generate Illumina-compatible libraries of sequences close to the 3'-end of poly(A) RNA. Sequencing was performed using a NextSeq 500 (Illumina), producing an average of $5 \times 10^{6}$ reads/sample (single-end, $75 \mathrm{bp}$ ).

Bioinformatics analysis of transcriptomic data generated on the in vitro model.

Reads from RNA-sequencing were subjected to quality check and trimming using the FastqQC and BBduk tools and to alignment using the STAR method. The Phread quality score was greater than 20 , and the percentage of alignment along the reference genome was higher than $80 \%$ along all the samples. Reads were aligned along genes using the HTseq count tool. Counts matrix was normalized with the TMM method (EdgeR). Gene expression TMM matrix counted a total of 17,650 genes for each of 132 samples. Principal Component Analysis (PCA) was performed using Variance Stabilizing Transformation (VST, DESeq2) on all genes under investigation. The single sample GSEA was performed starting from the normalized counts matrix with Hallmark collection 
gene set. For this analysis, a new gene expression matrix was defined, considering the mean expression of replicates (matrix 17.650x44). In the ssGSEA algorithm, averaged replicates were considered one by one. A score for each signature for each sample was calculated determining an absolute values score matrix (signature $\mathrm{x}$ samples). The score matrix was normalized considering the absolute value of the maximum value and the absolute value of the minimum one. The normalized matrix was plotted in a heatmap. The relative contribution of each variable and multiple combined effects was evaluated by supervised analysis at three levels of increasing complexity, as reported in the Supplementary information section.

Bioinformatics analysis of transcriptomic data generated on ex vivo samples.

Ex vivo data were analyzed with the same protocol by comparing pathological and control samples. Signatures generated by in vitro and ex vivo datasets were compared by using a multi-step method that we have developed. Pathway enrichment in the pathological samples was analyzed by Metascape online tool, considering DEGs in nephrectomy ex vivo data ( $\operatorname{logFC} \geq 1$ and $\mathrm{p}$-value $\leq$ 0.01). Only pathways with a $-\log _{10}$ (p-value) that exceeds the 1.3 threshold value were selected and represented by bar plots. Pre-ranked GSEA were analyzed by ranking ex vivo data on logFC, using in vitro data as input gene set signature. For each differential analysis a signature was obtained and the overlap between ex vivo and in vitro data was tested. Only in vitro signatures significantly enriched and overlapped with ex vivo signature were selected. See the Supplementary information section for a detailed description of pre-ranked GSEA and gene overlap analysis.

\section{Mathematical modeling.}

In brief, an ordinary differential equation model was constructed describing the dynamics of activated $\mathrm{M} \varphi$ and fibroblasts. The equations consist of terms related to the in vitro experimental observations and biological assumptions: 
A1. Proinflammatory $\mathrm{M} \varphi$ are recruited proportionally to the inflammation level.

A2. Profibrotic $\mathrm{M} \varphi$ proliferate in the presence of $\mathrm{Fb}$, in hypoxic and proinflammatory environments, while in normoxic environments, they become senescent (A, B).

$\mathrm{A} 3 . \mathrm{Fb}$ become activated and promote cell growth in the presence of $\mathrm{M} \varphi$ in hypoxic conditions. A4. All cell types become deactivated at a constant rate.

A5. Due to the short phenotypic switch rates compared to the system's characteristic time scale (inverse $\mathrm{M} \varphi$ deactivation rate), $\mathrm{M} \varphi$ were assumed to be in equilibrium with respect to their phenotypic switch.

Under these assumptions, we define the following system of ordinary differential equations:

$$
\begin{gathered}
\dot{M}_{1}=l Y-E_{1,2}-d_{1} M_{1}, \\
\dot{M}_{2}=r H \frac{M_{2} F\left(Y+Y_{0}\right)}{M_{2}+K}+E_{1,2}-d_{2} M_{2}, \\
\dot{F}=g H M_{2} F\left(F_{0}-F\right)-d_{3} F,
\end{gathered}
$$

where $M_{1}$ represents the number of proinflammatory $\mathrm{M} \varphi, M_{2}$ the number of profibrotic $\mathrm{M} \varphi, F$ the number of activated $\mathrm{Fb}, Y$ the inflammation level, $H$ the hypoxia level, $l$ the level of macrophage recruitment due to local inflammation, $d_{1}$ the death rate of proinflammatory $\mathrm{M} \varphi, r$ the profibrotic macrophage proliferation rate, $Y_{0}$ a reference level of inflammation, $K$ the base inhibition of profibrotic macrophage proliferation, $d_{2}$ the death rate of profibrotic $M \varphi, g$ the activation rate of $\mathrm{Fb}, F_{0}$ the mean maximum number of $\mathrm{Fb}$ in fibrosis, and $d_{3}$ the death rate of $\mathrm{Fb}$. Phenotypic switch between profibrotic and proinflammatory $\mathrm{M} \varphi$ was considered via the switching term:

$$
E_{1,2}=-k_{1 \rightarrow 2} M_{1}+k_{2 \rightarrow 1} M_{2} Y,
$$

where $k_{1 \rightarrow 2}$ and $k_{2 \rightarrow 1}$ are the switching rates from proinflammatory to profibrotic phenotypes and vice versa.

Due to the short phenotypic switch rates compared to the rates of cell proliferation and deactivation, $\mathrm{M} \varphi$ were assumed to be in equilibrium with respect to phenotypic switch. Assumption A5 allow us to link the number of profibrotic and proinflammatory $\mathrm{M} \varphi$ as: 


$$
Y=\lambda^{-1} \frac{M_{1}}{M_{2}}
$$

where $\lambda=\frac{k_{2 \rightarrow 1}}{k_{1 \rightarrow 2}}$ is the ratio of the switching rates. Using this expression, we can combine the equations for both macrophage phenotypes into a single equation for the total macrophage number, $M=M_{1}+M_{2}$. Further non-dimensionalization reduces the system to two equations:

$$
\begin{gathered}
\dot{m}=y-m \frac{\lambda y+\delta_{1}}{1+\lambda y}+\alpha H \frac{(y+1) m}{m+1+\lambda y} f, \\
\dot{f}=\beta H \frac{m}{1+\lambda y} f(1-f)-\delta_{2} f,
\end{gathered}
$$

where $m$ and $f$ are the non-dimensional macrophage and fibroblast populations, respectively, $y$ represents the non-dimensional inflammation, $\delta_{1}$ is related to the ratio of profibrotic to proinflammatory macrophage deactivation rates, $\delta_{2}$ is the ratio of fibroblast to proinflammatory deactivation rates, $\alpha$ is proportional to the profibrotic macrophage proliferation rate, and $\beta$ is proportional to the fibroblast proliferation rate. All quantities are non-dimensional.

The reduced model eqs $(1,2)$ has a similar structure to the one developed by Adler et al. [24] when the cytokines PDGF and CSF1 are assumed to be in a quasi-steady state. However, the activation rates of the two cell populations are modified by the impact of inflammation and hypoxia.

Under normoxic conditions $(H=0)$, there is a single stable steady state:

$$
\begin{array}{r}
m=\frac{y(1+\lambda y)}{\lambda y+\delta_{1}}, \\
f=0 . \quad(4)
\end{array}
$$

In hypoxia, this steady state exists along with two additional steady states. Linear stability analysis reveals that the steady state common to hypoxic and normoxic conditions (the "healthy" steady state) is a stable node as long as:

$$
H<\frac{\delta_{2}}{\beta}\left(\lambda+\frac{\delta_{1}}{y}\right)
$$

or written in terms of the inflammation level, which defines a critical inflammation, above which the system cannot return to the healthy state: 


$$
y<y_{c r}=\frac{\delta_{1}}{\frac{\beta}{\delta_{2}} H-\lambda} .
$$

It should be noted that, in the limit of very high inflammation $\left(\delta_{1} \ll \lambda\right)$, stability of the healthy state is still possible, as the stability condition reduces to:

$$
H<\frac{\lambda \delta_{2}}{\beta}
$$

One of the two steady states found only in hypoxic conditions is numerically found to correspond to a saddle point, while the other can be a stable or unstable node ("fibrotic" steady state) with $f \approx$ 1and $m \gg 1$. The inflammation-hypoxia space, see Fig. 10b, can be partitioned into three regions depending on the stability of the two nodes: I) the healthy state is stable, the fibrotic state is unstable; II) both states are stable; III) the healthy steady state is unstable, the fibrotic state is stable.

\section{Statistical analysis.}

Statistical analysis was performed using Prism version 7.0 (GraphPad software). Comparisons were calculated by two-way ANOVA test applying Sidak's multiple comparisons correction. The level of statistically significant difference was defined as $\mathrm{p} \leq 0.05$. 


\section{REFERENCES}

1. Wynn, T.A., Cellular and molecular mechanisms of fibrosis. J Pathol 214(2): p. 199-210 (2008).

2. Eddy, A.A., Overview of the cellular and molecular basis of kidney fibrosis. Kidney Int Suppl 4(1): p. 2-8 (2014).

3. Sato, M., et al., Targeted disruption of TGF-beta1/Smad3 signaling protects against renal tubulointerstitial fibrosis induced by unilateral ureteral obstruction. J Clin Invest 112(10): p. 1486-94 (2003).

4. Ueno, M., et al., Hypoxia-inducible factor- $1 \alpha$ mediates TGF- $\beta$-induced PAI- 1 production in alveolar macrophages in pulmonary fibrosis.Am J Physiol Lung Cell Mol Physiol 300(5): p. L740-52 (2011).

5. Koh, T.J. and L.A. DiPietro, Inflammation and wound healing: the role of the macrophage. Expert Rev Mol Med 13: p. e23 (2011).

6. Grande, M.T. and J.M. López-Novoa, Fibroblast activation and myofibroblast generation in obstructive nephropathy. Nat Rev Nephrol 5(6): p. 319-28 (2009).

7. Norman, J.T., I.M. Clark, and P.L. Garcia, Hypoxia promotes fibrogenesis in human renal fibroblasts. Kidney Int 58(6): p. 2351-66 (2000).

8. Nangaku, M. and K.U. Eckardt, Hypoxia and the HIF system in kidney disease. J Mol Med (Berl) 85(12): p. 1325-30 (2007).

9. Tanaka, T. and M. Nangaku, Angiogenesis and hypoxia in the kidney. Nat Rev Nephrol 9(4): p. 211-22 (2013). 
10. Li, M.O., et al., Transforming growth factor-beta regulation of immune responses. Annu Rev Immunol 24: p. 99-146 (2006).

11. Meran, S. and R. Steadman, Fibroblasts and myofibroblasts in renal fibrosis. Int J Exp Pathol 92(3): p. 158-67 (2011).

12. Cao, Q., D.C. Harris, and Y. Wang, Macrophages in kidney injury, inflammation, and fibrosis. Physiology (Bethesda) 30(3): p. 183-94 (2015).

13. Guiteras, R., et al., Macrophage overexpressing NGAL ameliorated kidney fibrosis in the UUO mice model. Cell Physiol Biochem 42(5): p. 1945-1960 (2017).

14. Bergler, T., et al., Infiltration of macrophages correlates with severity of allograft rejection and outcome in human kidney transplantation. PLoS One 11(6): p. e0156900 (2016).

15. Shen, B., et al., Macrophages regulate renal fibrosis through modulating TGF $\beta$ superfamily signaling. Inflammation 37(6): p. 2076-84 (2014).

16. Toki, D., et al., The role of macrophages in the development of human renal allograft fibrosis in the first year after transplantation. Am J Transplant 14(9): p. 2126-36 (2014).

17. Han, Y., Role of macrophages in the fibrotic phase of rat crescentic glomerulonephritis. Am J Physiol Renal Physiol 304(8): p.F1043-53 (2013).

18. Novak, M.L. and T.J. Koh, Phenotypic transitions of macrophages orchestrate tissue repair. Am J Pathol 183(5): p. 1352-1363 (2013).

19. Meng, X.M., et al., Macrophage phenotype in kidney injury and repair. Kidney Dis (Basel) 1(2): p. 138-46 (2015). 
20. Wynn, T.A. and K.M. Vannella, Macrophages in tissue repair, regeneration, and fibrosis. Immunity 44(3): p. 450-462 (2016).

21. Tang, P. M., Macrophages: versatile players in renal inflammation and fibrosis. Nat Rev Nephrol 15(3): p. 144-158 (2019).

22. Gieseck, R.L, et al., Type 2 immunity in tissue repair and fibrosis. Nat Rev Immunol 18(1): p.62-76 (2018).

23. Koh, T.J. and L.A. DiPietro, Inflammation and wound healing: the role of the macrophage. Expert Rev Mol Med 13: p. e23 (2011).

24. Adler,M., Principles of cell circuits for tissue repair and fibrosis. iScience 23(2):p.100841 (2020).

25. Limandjaja, G. C., et al., The keloid disorder: heterogeneity, histopathology, mechanisms and models. Front Cell Dev Biol 8: p.360 (2020).

26. Nathan, C. and A. Ding, Nonresolving inflammation. Cell 140(6): p. 871-82 (2010).

27. Medzhitov, R., et al., Origin and physiological roles of inflammation. Nature 454(7203): p. 428-35 (2008).

28. Martinez, F.O., et al., Genetic programs expressed in resting and IL-4 alternatively activated mouse and human macrophages: similarities and differences. Blood 121(9): p. e57-69 (2013).

29. Distler, J.H., et al., Hypoxia-induced increase in the production of extracellular matrix proteins in systemic sclerosis. Arthritis Rheum 56(12): p. 4203-15 (2007). 
30. Burke, B., et al., Hypoxia-induced gene expression in human macrophages: implications for ischemic tissues and hypoxia-regulated gene therapy. Am J Pathol 163(4): p. 1233-43 (2003).

31. Fuhrmann, D. C., et al., Chronic hypoxia alters mitochondrial composition in human macrophages. Biochim Biophys Acta 1834(12): p.2750-60 (2013).

32. Zhou, X., et al., Circuit design features of a stable two-cell system. Cell 172(4): p. 744757.e17 (2018).

33. Foxman, E. F., et al., Multistep navigation and the combinatorial control of leukocyte chemotaxis. J Cell Biol 139(5):1349-60 (1997).

34. Francis, L., Butcher, E., C., Modeling the role of homologous receptor desensitization in cell gradient sensing J Immunol 181(12):8335-43 (2008).

35. Mantovani, A., et al., Macrophage plasticity and polarization in tissue repair and remodelling. J Pathol 229(2): p. 176-85 (2013).

36. Mornata, F., et al., Reciprocal interference between the NRF2 and LPS signaling pathways on the immune-metabolic phenotype of peritoneal macrophages. Pharmacol Res Perspect e00638 (2020).

37. Wynn, T.A. and L. Barron, Macrophages: master regulators of inflammation and fibrosis. Semin Liver Dis 30(3): p. 245-57 (2010).

38. Curtale, G., et al., Multi-step regulation of the TLR4 pathway by the miR-125a 99b let-7e cluster. Front Immunol 9: p.2037 (2018). 
39. Tedesco, S., et al., Phenotypic activation and pharmacological outcomes of spontaneously differentiated human monocyte-derived macrophages. Immunobiology 220(5): p.545-54 (2015).

40. Merveille, O., et al., An automatic framework for fusing information from differently stained consecutive digital whole slide images: A case study in renal histology. Comput Methods Programs Biomed 208:106157 (2021).

41. Schaadt N. S., et al., Graph-based description of tertiary lymphoid organs at single-cell level. PLoS Comput Biol 16(2): p. e1007385 (2020). 


\section{ACKNOWLEDGEMENTS}

This study has been supported by the ERACoSysMed initiative as part of the European Union's Horizon 2020 Framework Program, with co-funding by the German Ministry of Research and Education (FKZ031L-0085A-SYSMIFTA, FKZ01ZX1710A-MicMode-I2T, and FKZ01ZX1608ASYSIMIT) and the Italian Ministry of Health (FKZ031L-0085A-SYSMIFTA). JMNS acknowledges support from the PAPIIT-UNAM (IA104821). The authors acknowledge excellent technical assistance in tissue processing and multiplexed immunohistochemistry by Nicole Kroenke, clinical data management by Irina Scheffner, and support in data export and management by Dr. Nadine Sarah Schaadt.

\section{AUTHOR CONTRIBUTIONS}

ES performed cell biology and RNAseq experiments and developed all figures but 6 and 10. AC analyzed RNAseq experiments. JMNS performed the mathematical analysis of the model. JW performed large-scale contextual image analysis and produced corresponding figures. RC contributed to analyze RNAseq experiments. AR performed the simulations of the model. VV performed laser capture microdissection, performed and analyzed multiplexed immunohistochemistry by multispectral image analysis, and produced images for figures 6 and 10 . JS retrieved archival paraffin-embedded tissue samples and performed laser capture microdissection. WG identified relevant clinical cohorts and individual cases and retrieved clinical information and follow-up data. HH supervised and designed the mathematical model, produced images for figure 10, and wrote the manuscript. FF designed the laser capture/RNAseq and immunohistochemical studies in kidney samples, coordinated funding for the SYSMIFTA and SYSIMIT research consortia, produced images for figures 6, 10 and S6, and wrote the manuscript. ML designed the cell biology and RNAseq experiments and wrote the manuscript. 


\section{COMPETING INTERESTS}

Authors have no competing interests to disclaim. 


\section{FIGURES}

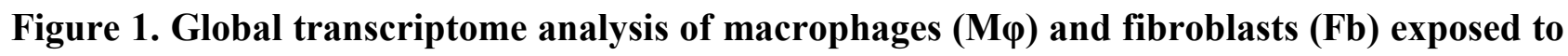
distinct immune, metabolic and culture conditions.

a) PCA on all samples indicates that the 2 components describing most of the system variability were represented by the cell type and inflammation (73\% - PC1 and 15\% - PC2, respectively, in the upper panel). Other source of variability were not detectable (see PC3 and PC4 in the bottom panel). For sample codes refer to Figure1b and S1a.

b) Single sample GSEA (ssGSEA) on the hallmark database of enriched gene ontologies. Labels indicate 44 sample conditions (columns) and hallmark categories enriched (rows). Samples were grouped by cell type $(\mathrm{M} \varphi$ or $\mathrm{Fb})$, cellular interaction (CC or $\mathrm{SC})$, immune cues $(0$ or I or $\mathrm{F})$ and metabolic cue ( $\mathrm{H}$ or $\mathrm{N}) .(\mathrm{M} \varphi$ and $\mathrm{Fb}$ in different shades of orange or blue, respectively). Different shape and color indicate different sample conditions: squares $=$ resting $(0)$, circles $=$ proinflammatory $(\mathrm{I})$ and triangles = profibrotic $(\mathrm{F})$; empty and full shapes are related to single culture (SC) and coculture (CC), respectively; different shade of color are related to time (4 or $24 \mathrm{~h}$ ) and oxygen status $(\mathrm{N}=$ normoxia, $\mathrm{H}=$ hypoxia $)$.

c-h) Quantification of differentially expressed genes (DEGs; FDR $\leq 0.05)$ in $\mathrm{M} \varphi$ and Fb depending on immune cues, hypoxia, or cellular interaction. Panel c) reports the effect of immune cues comparing proinflammatory or profibrotic $\mathrm{M} \varphi$ to resting $\mathrm{M} \varphi(\mathrm{MIvsM} 0=$ red bars, $\mathrm{MFvsM0}=$ green bars, respectively) in SC or CC (empty and full bars, respectively) at 4 or $24 \mathrm{~h}$ of normoxia (4N or $24 \mathrm{~N})$ or hypoxia $(4 \mathrm{H}$ or $24 \mathrm{H})$. Panel d) reports the effect of hypoxia on $\mathrm{M} \varphi$ comparing hypoxic to normoxic $\mathrm{M} \varphi$ in resting (MHvsM0/N = grey bars), proinflammatory $(\mathrm{MI} / \mathrm{HvsMI} / \mathrm{N}=$ red bars) and profibrotic $(\mathrm{MF} / \mathrm{HvsMF} / \mathrm{N}=$ green bars) conditions, in $\mathrm{SC}$ or $\mathrm{CC}$ (empty and full bars, respectively), both at 4 or $24 \mathrm{~h}$. Panel e) reports the effect of $\mathrm{Fb}$ on $\mathrm{M} \varphi$ comparing cocultivated to single cultivated $\mathrm{M} \varphi$ in resting $(\mathrm{M} 0 / \mathrm{CCvsM} 0 / \mathrm{SC}=$ grey bars $)$, proinflammatory $(\mathrm{MI} / \mathrm{CCvsMI} / \mathrm{SC}=$ 
red bars) and profibrotic $(\mathrm{MF} / \mathrm{CCvsMF} / \mathrm{SC}=$ green bars) conditions at 4 or $24 \mathrm{~h}$ of normoxia $(4 \mathrm{~N}$ or $24 \mathrm{~N}$ ) or hypoxia $(4 \mathrm{H}$ or $24 \mathrm{H})$. Panels f-h report the same comparisons of panels c-e, respectively, but performed on $\mathrm{Fb}$.

\section{Figure 2. Role of inflammation on the global biological response.}

a,d) Schematic representation of the multi-level approach to study the effect of inflammation on $\mathrm{M} \varphi$ (panels a-c) and $\mathrm{Fb}$ (panels d-f) in different metabolic and culture conditions. $1^{\text {st }}$ level compares LPS +IFN $\gamma$ stimulated cells to resting cells in four different conditions: normoxic and single culture (comparison $A_{M} / A_{F}$ in pink), hypoxic and single culture $\left(B_{M} / B_{F}\right.$ in blue), normoxic and coculture $\left(\mathrm{C}_{\mathrm{M}} / \mathrm{C}_{\mathrm{F}}\right.$ in green), hypoxic and coculture ( $\mathrm{D}_{\mathrm{M}} / \mathrm{D}_{\mathrm{F}}$ in yellow). $2^{\text {nd }}$ level (double arrows) compares hypoxic to normoxic treatment (AvsB and CvsD), $3^{\text {rd }}$ level (triple arrows) compares coculture to single culture conditions $[(\mathrm{A}+\mathrm{B}) \mathrm{vs}(\mathrm{C}+\mathrm{D})]$.

b, e) Venn diagrams show the distribution of DEGs in each $1^{\text {st }}$ level comparison in $\mathrm{M} \varphi(\mathrm{b})$ and $\mathrm{Fb}$ (e).

$\mathrm{d}, \mathrm{f})$ Pathways enrichment analysis in $\mathrm{M \varphi}(\mathrm{d})$ and $\mathrm{Fb}$ (f) comparisons. Columns represent $1^{\text {st }}$ level of comparisons (A-B-C-D), rows report pathways significantly modulated ( $\mid \mathrm{z}$-score $\mid \geq 2$ ) in at least one comparison. Color intensity bar indicates the level of positive (in red) or negative (in blue) enrichment; dots appear only when pathways are significantly enriched $(\bullet \mathrm{z}$-score $>2 ; \bullet \bullet$ z-score $>$ 4; • z-score $<-2 ; \bullet$ z-score $<-4)$; grey indicates no modulation. See also Fig. S2.

\section{Figure 3. Role of adaptive Th2 immune response (IL-4) on the global biological response.}

a, d) Schematic representation of the multi-level approach to study the effect of Th2 cytokine on $\mathrm{M} \varphi$ (panels a-c) and $\mathrm{Fb}$ (panels d-f) in different metabolic and culture conditions. $1^{\text {st }}$ level compares IL-4 stimulated cells to resting cells in four different conditions: normoxic and single culture (comparison $A_{M} / A_{F}$ in pink), hypoxic and single culture $\left(\mathrm{B}_{M} / B_{F}\right.$ in blue), normoxic and 
coculture $\left(\mathrm{C}_{\mathrm{M}} / \mathrm{C}_{\mathrm{F}}\right.$ in green $)$, hypoxic and coculture $\left(\mathrm{D}_{\mathrm{M}} / \mathrm{D}_{\mathrm{F}}\right.$ in yellow). $2^{\text {nd }}$ level (double arrows) compares hypoxic to normoxic treatment (AvsB and CvsD) and $3^{\text {rd }}$ level (triple arrows) compares coculture to single culture conditions $[(\mathrm{A}+\mathrm{B}) \mathrm{vs}(\mathrm{C}+\mathrm{D})]$.

b, e) Venn diagrams with DEGs distribution in each $1^{\text {st }}$ level comparison in $\mathrm{M} \varphi(\mathrm{b})$ and $\mathrm{Fb}(\mathrm{e})$.

d, f) Pathways enrichment analysis in $\mathrm{M \varphi}(\mathrm{d})$ and $\mathrm{Fb}$ (f) comparisons. Columns represent $1^{\text {st }}$ level comparisons (A-B-C-D), rows report pathways significantly modulated (|z-score $\mid \geq 2$ ) in at least one comparison. Bar color intensity indicates the level of positive (red) or negative (blue) enrichment; dots appear only when pathways are significantly enriched $(\bullet \mathrm{z}$-score $>2 ; \bullet \bullet \mathrm{z}$-score $>4$; $\bullet$ Z-score $<$ 2 ; $\bullet$ z-score $<-4)$; grey indicates no modulation. See also Fig. S3.

\section{Figure 4. Role of hypoxia on the global biological response.}

a, d) Schematic representation of the multi-level approach to study the effect of hypoxia on $\mathrm{M} \varphi$ (panels a-c) and $\mathrm{Fb}$ (panels $\mathrm{d}-\mathrm{f}$ ) in different immune and culture conditions. $1^{\text {st }}$ level compares hypoxic cells to normoxic cells in four different conditions: resting and single culture (comparison $A_{M} / A_{F}$ in pink), resting and coculture $\left(B_{M} / B_{F}\right.$ in blue $), L P S+I F N \gamma$ and single culture $\left(C_{M} / C_{F}\right.$ in green), LPS + IFN $\gamma$ and coculture $\left(\mathrm{D}_{\mathrm{M}} / \mathrm{D}_{\mathrm{F}}\right.$ in yellow); $2^{\text {nd }}$ level (double arrows) compares coculture to single culture conditions (AvsB and $\mathrm{CvsD}$ ); $3^{\text {rd }}$ level (triple arrows) compares resting to proinflammatory conditions $[(\mathrm{A}+\mathrm{B}) \mathrm{vs}(\mathrm{C}+\mathrm{D})]$.

b,e) Venn diagrams with DEGs distribution in each $1^{\text {st }}$ level comparison in $\mathrm{M} \varphi(\mathrm{b})$ and $\mathrm{Fb}(\mathrm{e})$.

c, f) Pathways enrichment analysis in $\mathrm{M} \varphi(\mathrm{c})$ and $\mathrm{Fb}$ (f) comparisons. Columns represent $1^{\text {st }}$ level analysis (A-B-C-D), rows report pathways significantly modulated (|z-score $\mid \geq 2$ ) in at least one comparison. Color intensity bar indicates the level of positive (in red) or negative (in blue) enrichment; dots appear only when pathways are significantly enriched $(\bullet \mathrm{z}$-score $>2 ; \bullet \mathrm{z}$-score $>4$;

- z-score $<-2 ; \bullet$ z-score $<-4$ ); grey indicates no modulation. See also Fig. S4. 


\section{Figure 5. Role of cellular interplay on the global biological response.}

a, d) Schematic representation of the multi-level approach to study the effect of $\mathrm{Fb}$ on $\mathrm{M} \varphi$ (panels a-c) and of $\mathrm{M} \varphi$ on $\mathrm{Fb}$ (panels d-f) in a tissue-like setting with different metabolic and immune conditions. $1^{\text {st }}$ level compares cocultivated cells to single cultivated cells in four different conditions: in normoxic and resting (comparison $A_{M} / A_{F}$ in pink), in hypoxic and resting $\left(B_{M} / B_{F}\right.$ in blue), in normoxic and inflamed $\left(\mathrm{C}_{\mathrm{M}} / \mathrm{C}_{\mathrm{F}}\right.$ in green $)$, in hypoxic and inflamed ( $\mathrm{D}_{\mathrm{M}} / \mathrm{D}_{\mathrm{F}}$ in yellow). $2^{\text {nd }}$ level (double arrows) compares normoxic to hypoxic environments (AvsB and CvsD) and $3^{\text {rd }}$ level (triple arrows) compares resting to proinflammatory conditions $[(\mathrm{A}+\mathrm{B}) \mathrm{vs}(\mathrm{C}+\mathrm{D})]$.

b, e) Venn diagrams show the distribution of DEGs in each $1^{\text {st }}$ level comparison in $\mathrm{M} \varphi$ (b) and $\mathrm{Fb}$ (e).

$\mathrm{d}, \mathrm{f}$ ) Pathways enrichment analysis in $\mathrm{M \varphi}(\mathrm{d})$ and $\mathrm{Fb}(\mathrm{f})$ comparisons. Columns represent $1^{\text {st }}$ level of comparisons (A-B-C-D), rows report pathways significantly modulated $(|z-s c o r e| \geq 2)$ in at least one comparison. Color intensity bar indicates the level of positive (in red) or negative (in blue) enrichment; dots appear only when pathways are significantly enriched $(\bullet \mathrm{z}$-score $>2 ; \bullet$ z-score $>$ 4; • Z-score $<-2 ; \bullet$ z-score $<-4)$; grey indicates no modulation. See also Fig. S5.

\section{Figure 6. Different stages of fibrosis and heterogeneity of immune cell infiltrates characterizing distinct anatomical kidney regions.}

(a-c) Multiplexed immunohistochemistry of transplant nephrectomy samples in (a) acute cellmediated rejection (TCMR; BANFF type IIa), (b) chronic antibody-mediated rejection (ABMR; BANFF type III), and (c) active stage of fibrosis (ca. 90\% IF/TA; BANFF grade III) with signs of chronic rejection that make it difficult to differentiate between TCMR and ABMR. The left panel of (a-c) shows fluorescent whole slide scans for overview in tissue context. The right 3 panels show region-specific immune cell phenotyping in multiple fields of view after multispectral unmixing (see color code in right panel of a-c). Representative examples of the glomerular compartment (Glo) 
and the tubulointerstitial areas (IF/TA) are displayed. Co-existence of almost normal Glo and tubulointerstitial areas (a, left panel, arrows) and severely inflamed regions (a, left panel, asterisks) can be observed. Glomeruli were almost devoid of $\mathrm{M} \varphi$ with few exceptions (b, right panel, arrows). In glomeruli, $\mathrm{M} \varphi$ were not in direct neighborhood to $\mathrm{FAP}^{+}$myofibroblasts (Fig. 6c, right panel, arrows).

d) Cell density bar plot of different cell types: macrophages (CD68 $\left.{ }^{+} / \mathrm{CD} 206^{+}, \mathrm{CD}^{+} 8^{+} / \mathrm{CD} 206^{-}\right)$, Tlymphocytes $\left(\mathrm{CD}^{+}, \mathrm{CD}^{+}\right)$, B lymphocytes $\left(\mathrm{CD} 20^{+}\right)$and fibroblasts $\left(\mathrm{FAP}^{+}\right)$, corresponding to (c) in each anatomical compartment $($ glomeruli $=$ green; interstitium $=$ violet; surrounding $=$ orange; $*$ $\mathrm{p}<0.05, * * \mathrm{p}<0.01)$

(e) Sirius red staining (corresponding to c) showing co-existence of morphologically almost intact Glo and sclerotic glomeruli (sGlo), and tubulointerstitial areas ranging from severe (**) to moderate/low fibrosis (*). (f) IHC for FAP (corresponding to c), showing co-existence of almost normal Glo and areas of massive myofibroblast activation (sGlo), and tubulointerstitial areas ranging from severe $(* *)$ to moderate/low myofibroblast activation $(*)$. (g) Duplex immunohistochemistry (corresponding to c) confirmed the heterogeneous distribution of activated myofibroblasts $\left(\mathrm{FAP}^{+}\right)$, and alternatively activated $\mathrm{CD} 206^{+} \mathrm{M} \varphi$ in different anatomical regions. (h-j) Neighborhood analysis on multiplexed IHC. For each region, glomeruli (h), interstitium (i) and surrounding $(j)$ is evaluated on the average number of different cell types near $(7.5 \mu \mathrm{m})$ the type of interest (horizontal axis). Under each cell type its absolute number is reported.

Figure 7. Gene overlap analysis of ex vivo tubulointerstitial area from fibrotic kidneys with in vitro signatures.

a) PCA of ex vivo interstitium samples. Triangles indicate fibrotic kidneys, dot control kidneys.

b) Bar graph reports pathways significantly enriched in fibrotic vs control interstitium samples as calculated by Metascape software. 
c) Overlap analysis was performed comparing DEGs of ex vivo fibrotic vs control interstitium with DEGs of each in vitro comparison (which defined 78 corresponding signatures). Pie chart reveals that $49.9 \%$ of ex vivo signature is explained by at least one in vitro signature (in light green); $10.8 \%$ is specifically explained by only one in vitro signature and different colors are referred to the discriminating variable: inflammation in red, IL-4 stimulation in green, cell-cell interaction in blue, hypoxia in yellow. The remaining $39.3 \%$ of the ex vivo interstitium signature (633 genes) is not explained by in vitro model (in grey). Dot chart reports on the vertical axis 19 in vitro signatures enriched in ex vivo model and their relative contribution with unique expressed genes: white numbers in each dot represent relative percentages on the totality of explained and not explained genes. The central heatmap highlights the overlapping percentage of the same 19 in vitro signatures on the ex vivo signature. On the diagonal, numbers indicate the overlapping percentage of each signature; the other numbers explain the overlapping percentage shared between two in vitro and the ex vivo signatures. Color intensity indicates the overlapping percentage level referred to ex vivo signature. Arrows near each in vitro signature indicate the positive (red) or negative (blue) enrichment of the first term of in vitro comparison (see also Fig. S7). The heatmap on the right reports Metascape pathways in ex vivo comparison (panel b) with the percentage of enrichment given by each in vitro signature (on the left).

Figure 8. Gene overlap analysis of ex vivo glomeruli surrounding area from fibrotic kidneys with in vitro signatures.

a) PCA of ex vivo glomeruli surrounding area samples. Triangles indicate fibrotic kidneys, dot control samples.

b) Bar graph reports pathways significantly enriched in pathological vs control surrounding samples as calculated by Metascape software. 
c) Overlap analysis was performed comparing DEGs of ex vivo fibrotic vs control surrounding areas with DEGs of each in vitro comparison (which defined 78 corresponding signatures). Pie chart reveals that $52.7 \%$ of ex vivo signature is explained by at least one in vitro signature (in light green); $10.1 \%$ is specifically explained by only one in vitro signature and different colors are referred to the discriminating variable of in vitro signatures: inflammation in red, IL-4 stimulation in green, cellcell interaction in blue, hypoxia in yellow. The remaining $37.2 \%$ of the surrounding ex vivo signature (2,685 genes) is not explained by in vitro model (in grey). Dot chart reports on the vertical axis 24 in vitro signatures enriched in ex vivo model and their relative contribution with unique expressed genes: white numbers in each dot represent relative percentages on the totality of explained and not explained genes. The central heatmap highlights the overlapping percentage of the same 24 in vitro signatures. On the diagonal, numbers indicate the overlapping percentage of each signature; the other numbers explain the overlapping percentage shared between two in vitro and the ex vivo signatures. Color intensity indicates the overlapping percentage level referred to $e x$ vivo signature. Arrows near each in vitro signature indicate the positive (red) or negative (blue) enrichment of the first term of in vitro comparison (see also Fig. S7). The heatmap on the right reports Metascape pathways in ex vivo comparison (panel b) with the percentage of enrichment given by each in vitro signature (on the left). Orange stars highlight pathways that are specifically enriched in surrounding nephrectomies and are not shared with other renal districts (interstitium, glomeruli).

Figure 9. Gene overlap analysis of ex vivo glomeruli from fibrotic kidneys with in vitro signatures.

a) PCA of ex vivo glomeruli samples. Triangles indicate fibrotic kidneys, dot control samples.

b) Bar graph reports pathways significantly enriched in fibrotic vs control glomeruli samples as calculated by Metascape software. 
c) Overlap analysis was performed comparing DEGs of ex vivo fibrotic vs control glomeruli with DEGs of each in vitro comparison (which defined 78 corresponding signatures). Pie chart reveals that $42.3 \%$ of ex vivo signature is explained by at least one in vitro signature (in light green); $10.2 \%$ is specifically explained by only one in vitro signature and different colors are referred to the discriminating variable of in vitro signatures: inflammation in red, IL-4 stimulation in green, cellcell interaction in blue, hypoxia in yellow. The remaining $47.5 \%$ of the ex vivo glomeruli signature (255 genes) is not explained by in vitro model (in grey). Dot chart reports on the vertical axis 12 in vitro signatures enriched in ex vivo model and their relative contribution with unique expressed genes: white numbers in each dot represent relative percentages on the totality of explained and not explained genes. The central heatmap highlights the overlapping percentage of the same 12 in vitro signatures on the ex vivo signature. On the diagonal, numbers indicate the overlapping percentage of each signature; the other numbers explain the overlapping percentage shared between two in vitro and the ex vivo signatures. Color intensity indicates the overlapping percentage level referred to $e x$ vivo signature. Arrows near each in vitro signature indicate the positive (red) or negative (blue) enrichment of the first term of in vitro comparison. See also Fig. S7. The heatmap on the right reports Metascape pathways in ex vivo comparison (panel b) with the percentage of enrichment given by each in vitro signature (on the left). Green stars highlight pathways that are specifically enriched in glomeruli nephrectomies and are not shared with other renal districts (interstitium, surrounding area).

\section{Figure 10. Mathematical model based on in vitro data and exemplary clinical observations}

\section{reflecting the predicted outcomes.}

a) Schematic representation of $\mathrm{M} \varphi(\mathrm{MI}$ in red and $\mathrm{MF}$ in green) and $\mathrm{Fb}$ interactions under hypoxia (H) and inflammation (Y). 
b) Phase diagram of mathematical prediction. Three phases (I-II-II) were identified on different levels of inflammation (horizontal axis) and hypoxia (vertical axis). The red line between phases II and III is the separatrix.

c) Nine exemplary cases (case 1-9) reflecting the stable "Phase I": The renal function, represented by the glomerular filtration rate (GFR) over time after transplantation, can stay stable over many years, despite substantial infiltration by immune cells, including many macrophages, in a protocol biopsy taken around the time point $\sim 6$ months after transplantation. Pie charts describe the individual immune cell infiltrate (lymphocytes and $\mathrm{M} \varphi$ ) evaluated by quantitative digital pathology after multiplex immunohistochemistry. The percentage of immune cells (color code in the figure legend indicates the immunophenotype) in comparison to all nucleated cells in the biopsy as defined by DAPI nuclear staining (grey area in the pie charts) can massively vary between almost no inflammation (e.g., case 3, 8) and abundant infiltrates (e.g., cases 5, 6, 9). Nevertheless, the renal function stays stable over 2-5 years, indicating that the inflammatory process resolved to a nonfibrotic state in sufficiently large areas of the transplanted kidney.

d) Graphical depiction of in-silico simulations (left panels) show the solution trajectory and the predicted dynamics of $\mathrm{M} \varphi$ and $\mathrm{Fb}$ depending on time (horizontal axis) and cell fraction (vertical axis) for each of the three phases as predicted by the mathematical model in more detail. The right panel shows corresponding representative clinical constellations that confirmed the hypotheses generated by computer simulations, based on the exemplary cases 10 (unfavorable outcome with irreversible "hot" and "cold" fibrosis) and case 11 (favorable outcome despite "borderline" immune infiltration). The color legend indicates the different cell types as detected my multiplexed immunohistochemistry. Note the abundant CD206 ${ }^{+}$macrophages in the indicated biopsy of case 11 in "Phase II" in the surrounding area (upper image), inside the glomerulus (middle image) and in the interstitial compartment (bottom image), and in case 10 in "Phase III" the co-existence of areas with many CD206 macrophages ("hot" fibrosis) and low density of this phenotype ("cold" 
fibrosis) co-localized with myofibroblast activation ( $\mathrm{FAP}^{+}$cells) in the same biopsy as predicted by in-silico modeling. 
Figures
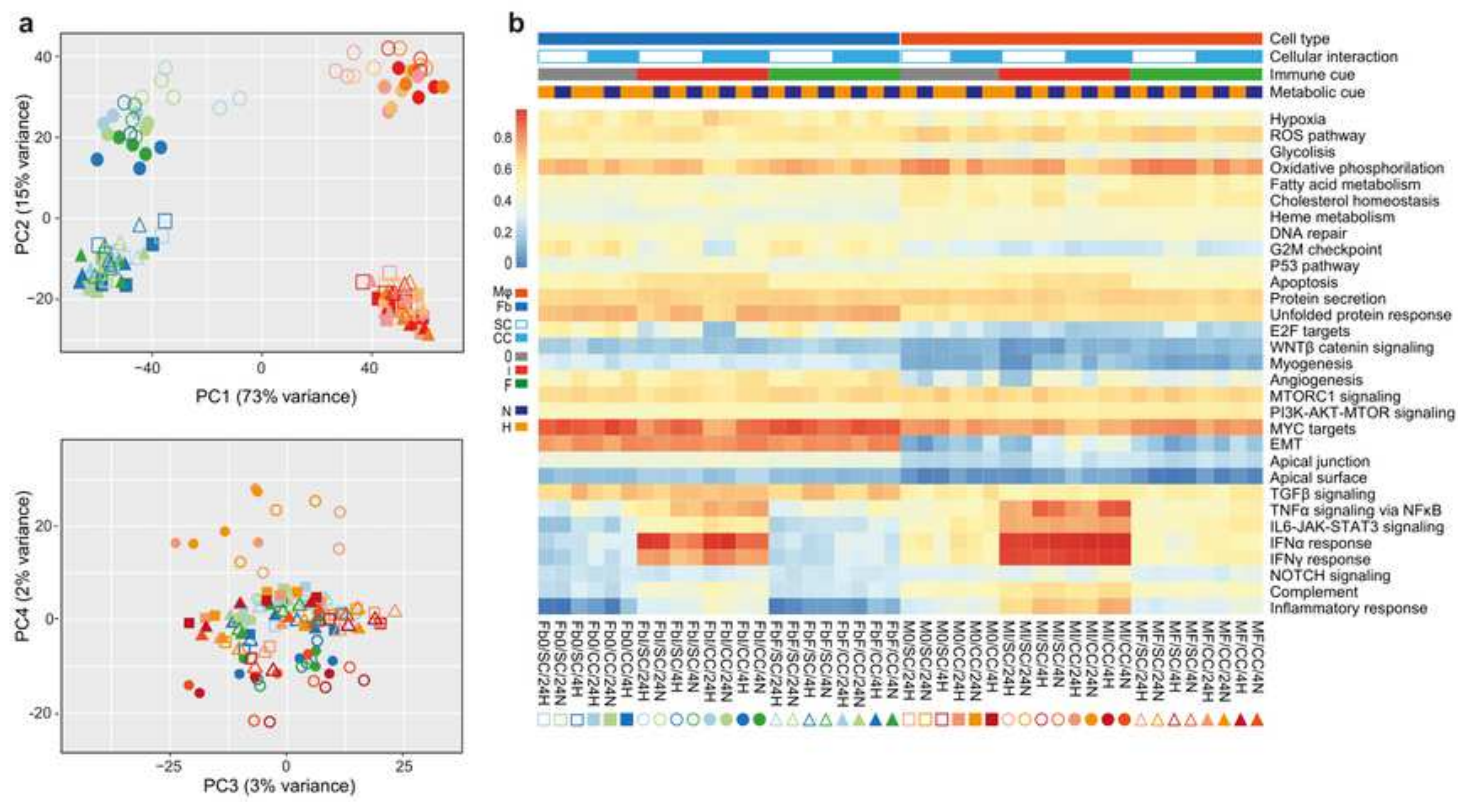

d

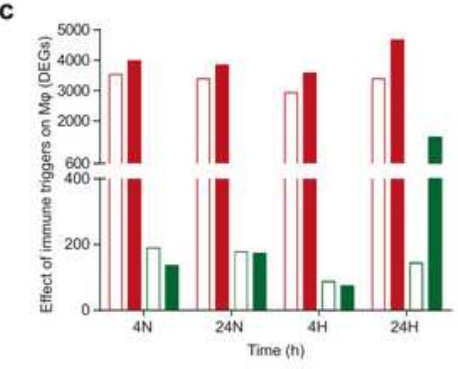

$\square$ MivsMosc $\square$ MivsMocc

f

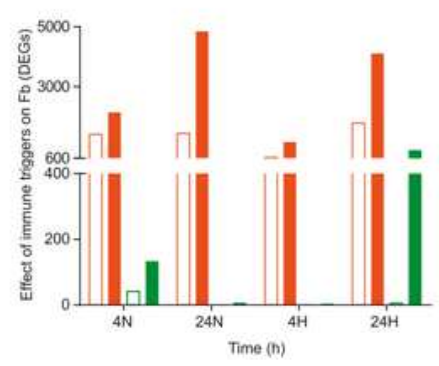

$\square$ FblvsFbosc $=$ FblvsFboCC $\square$ FbFvsFDOSC

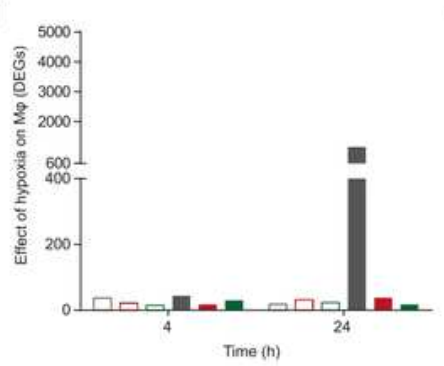

$\square$ MHvsMaN SC MHVSMO/NCC

$\square$ MIHUsMUINSC $=$ MLHUsMIINCC

g

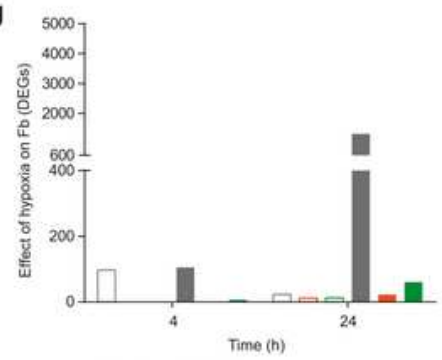

$\square$ FbHWSFONSC $=$ FbHWSFON CC

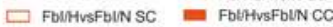
$\square$ FbF/HUSFbFINSC $=$ FbFHUSFbNCC e

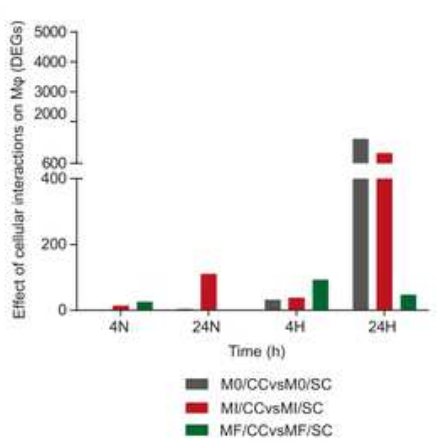

h

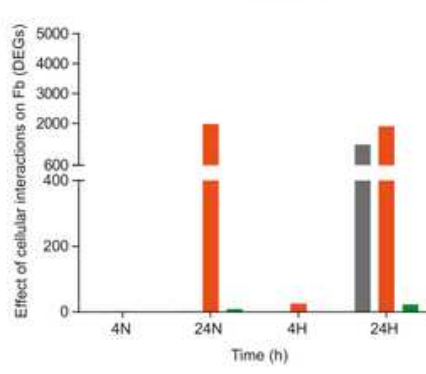

- Fboiccusfbolsc

mblicCusfousC

- FbFICCVSFDFISC

\section{Figure 1}

Global transcriptome analysis of macrophages $(\mathrm{M} \varphi)$ and fibroblasts $(\mathrm{Fb})$ exposed to distinct immune, metabolic and culture conditions. a) PCA on all samples indicates that the 2 components describing most of the system variability were represented by the cell type and inflammation (73\% - PC1 and 15\% - 
PC2, respectively, in the upper panel). Other source of variability were not detectable (see PC3 and PC4 in the bottom panel). For sample codes refer to Figure1b and S1a. b) Single sample GSEA (SSGSEA) on the hallmark database of enriched gene ontologies. Labels indicate 44 sample conditions (columns) and hallmark categories enriched (rows). Samples were grouped by cell type ( $M \varphi$ or Fb), cellular interaction (CC or $\mathrm{SC}$ ), immune cues ( 0 or I or $\mathrm{F}$ ) and metabolic cue ( $\mathrm{H}$ or $\mathrm{N})$. $(\mathrm{M} \varphi$ and $\mathrm{Fb}$ in different shades of orange or blue, respectively). Different shape and color indicate different sample conditions: squares = resting $(0)$, circles $=$ proinflammatory $(I)$ and triangles $=$ profibrotic $(F)$; empty and full shapes are related to single culture (SC) and coculture (CC), respectively; different shade of color are related to time (4 or 24h) and oxygen status ( $\mathrm{N}=$ normoxia, $\mathrm{H}=$ hypoxia). $\mathrm{c}-\mathrm{h}$ ) Quantification of differentially expressed genes (DEGs; FDR $\leq 0.05$ ) in $M \varphi$ and $F b$ depending on immune cues, hypoxia, or cellular interaction. Panel c) reports the effect of immune cues comparing proinflammatory or profibrotic $M \varphi$ to resting $M \varphi$ (MlvsM0= red bars, MFvsM0 = green bars, respectively) in SC or CC (empty and full bars, respectively) at 4 or $24 \mathrm{~h}$ of normoxia $(4 \mathrm{~N}$ or $24 \mathrm{~N}$ ) or hypoxia $(4 \mathrm{H}$ or $24 \mathrm{H})$. Panel d) reports the effect of hypoxia on $\mathrm{M} \varphi$ comparing hypoxic to normoxic $\mathrm{M} \varphi$ in resting ( $\mathrm{MHvsMO} / \mathrm{N}=$ grey bars), proinflammatory ( $\mathrm{Ml} / \mathrm{HvsMI} / \mathrm{N}=$ red bars) and profibrotic (MF/HvsMF/N = green bars) conditions, in SC or CC (empty and full bars, respectively), both at 4 or $24 \mathrm{~h}$. Panel e) reports the effect of $\mathrm{Fb}$ on $\mathrm{M} \varphi$ comparing cocultivated to single cultivated $\mathrm{M} \varphi$ in resting (MO/CCvsM0/SC = grey bars), proinflammatory $(\mathrm{MI} / \mathrm{CCVSMI} / \mathrm{SC}=$ red bars $)$ and profibrotic (MF/CCvsMF/SC $=$ green bars) conditions at 4 or $24 \mathrm{~h}$ of normoxia $(4 \mathrm{~N}$ or $24 \mathrm{~N})$ or hypoxia $(4 \mathrm{H}$ or $24 \mathrm{H})$. Panels $\mathrm{f}$-h report the same comparisons of panels c-e, respectively, but performed on Fb. 
a

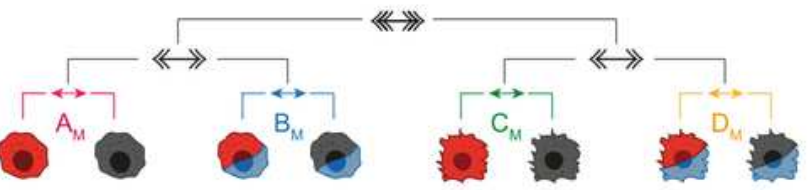

b

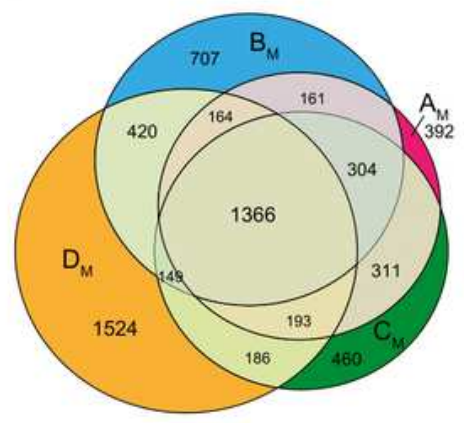

c

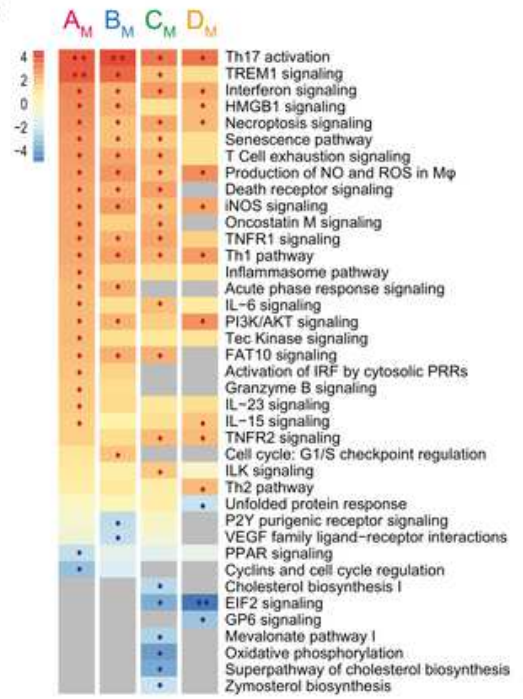

$$
\text { d }
$$

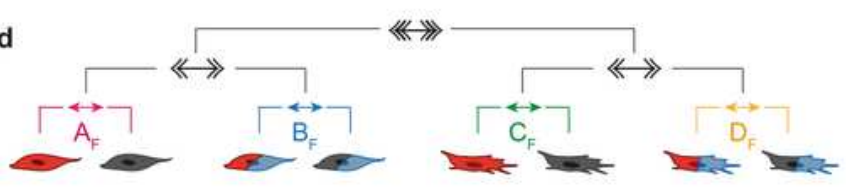

e
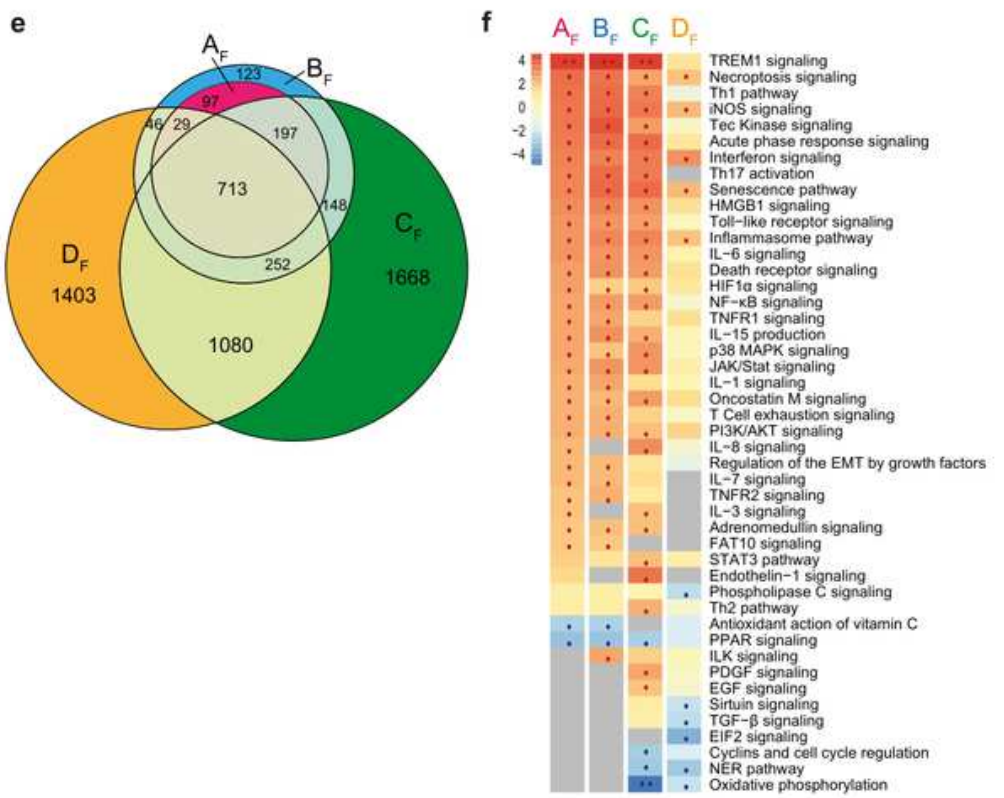

\section{Figure 2}

Role of inflammation on the global biological response. a,d) Schematic representation of the multi-level approach to study the effect of inflammation on $\mathrm{M} \varphi$ (panels a-c) and $\mathrm{Fb}$ (panels d-f) in different metabolic and culture conditions. 1st level compares LPS+IFNy stimulated cells to resting cells in four different conditions: normoxic and single culture (comparison AM/AF in pink), hypoxic and single culture (BM/BF in blue), normoxic and coculture (CM/CF in green), hypoxic and coculture (DM/DF in yellow). 2nd 
level (double arrows) compares hypoxic to normoxic treatment (AvsB and CvsD), 3rd level (triple arrows) compares coculture to single culture conditions $[(A+B) v s(C+D)] . b, e)$ Venn diagrams show the distribution of DEGs in each 1st level comparison in $M \varphi(b)$ and $F b(e) . d$, f) Pathways enrichment analysis in $M \varphi$ (d) and Fb (f) comparisons. Columns represent 1 st level of comparisons (A-B-C-D), rows report pathways significantly modulated ( $\mid z$-score| $\geq 2)$ in at least one comparison. Color intensity bar indicates the level of positive (in red) or negative (in blue) enrichment; dots appear only when pathways are significantly enriched $(\cdot z$-score $>2 ; \cdot \cdot z$-score $>4 ; \cdot z$-score $<-2 ; \cdot \cdot z$-score $<-4)$; grey indicates no modulation. See also Fig. S2. 
a

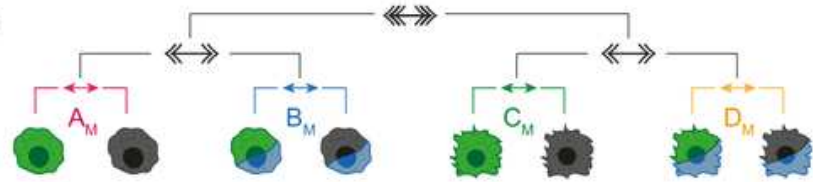

b

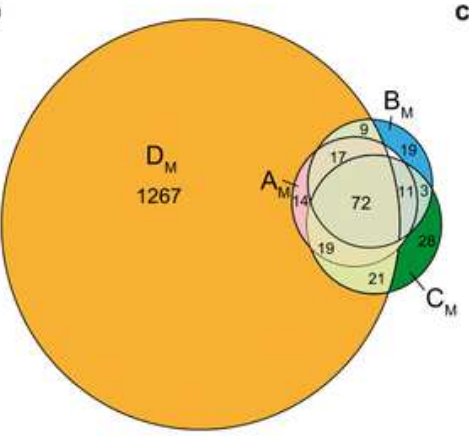

c $\quad A_{M} B_{M} C_{M} D_{M}$

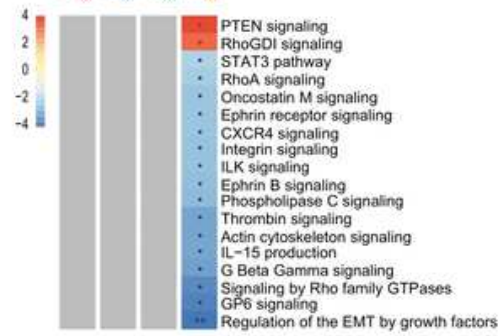

d

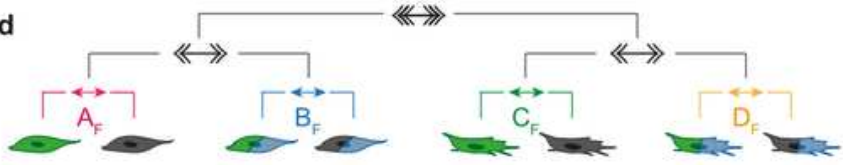

e f $\quad A_{F} B_{F} C_{F} D_{F}$
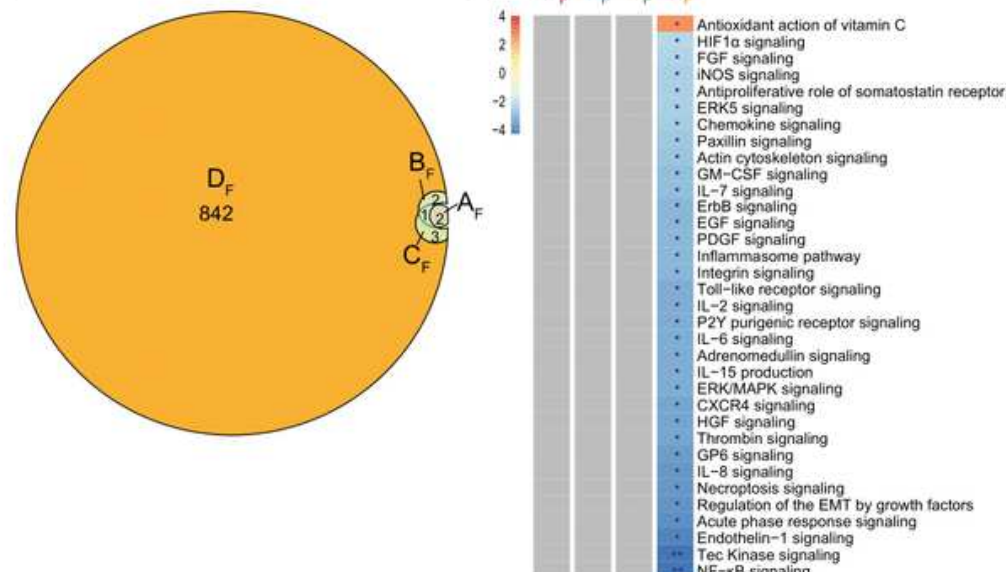

\section{Figure 3}

Role of adaptive Th2 immune response (IL-4) on the global biological response. a, d) Schematic representation of the multi-level approach to study the effect of Th2 cytokine on $M \varphi$ (panels a-c) and $\mathrm{Fb}$ (panels $\mathrm{d}-\mathrm{f}$ ) in different metabolic and culture conditions. 1st level compares IL-4 stimulated cells to resting cells in four different conditions: normoxic and single culture (comparison AM/AF in pink), hypoxic and single culture (BM/BF in blue), normoxic and coculture(CM/CF in green), hypoxic and 
coculture (DM/DF in yellow). 2nd level (double arrows) compares hypoxic to normoxic treatment (AvsB and $\mathrm{CvsD}$ ) and 3rd level (triple arrows) compares coculture to single culture conditions $[(A+B) v s(C+D)] . b$, e) Venn diagrams with DEGs distribution in each 1st level comparison in $M \varphi(b)$ and $F b(e) . d, f)$ Pathways enrichment analysis in $M \varphi$ (d) and $\mathrm{Fb}$ (f) comparisons. Columns represent 1stlevel comparisons (A-B-C-D), rows report pathways significantly modulated ( $\mid z$-score $\mid \geq 2)$ in at least one comparison. Bar color intensity indicates the level of positive (red) or negative (blue) enrichment; dots appear only when pathways are significantly enriched $(\cdot z$-score $>2 ; \cdot \cdot z$-score $>4$; $\cdot \mathbf{z}$-score $<-2 ; \cdot \cdot z$-score $<$ $-4)$; grey indicates no modulation. See also Fig. S3. 
a

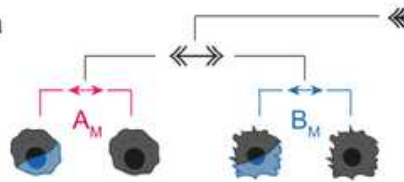
《«

b

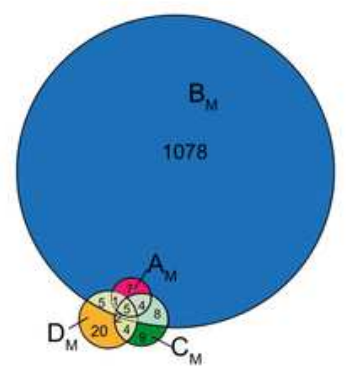

C

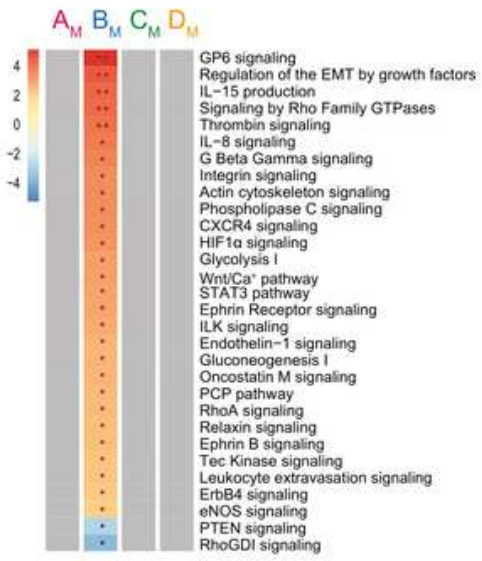

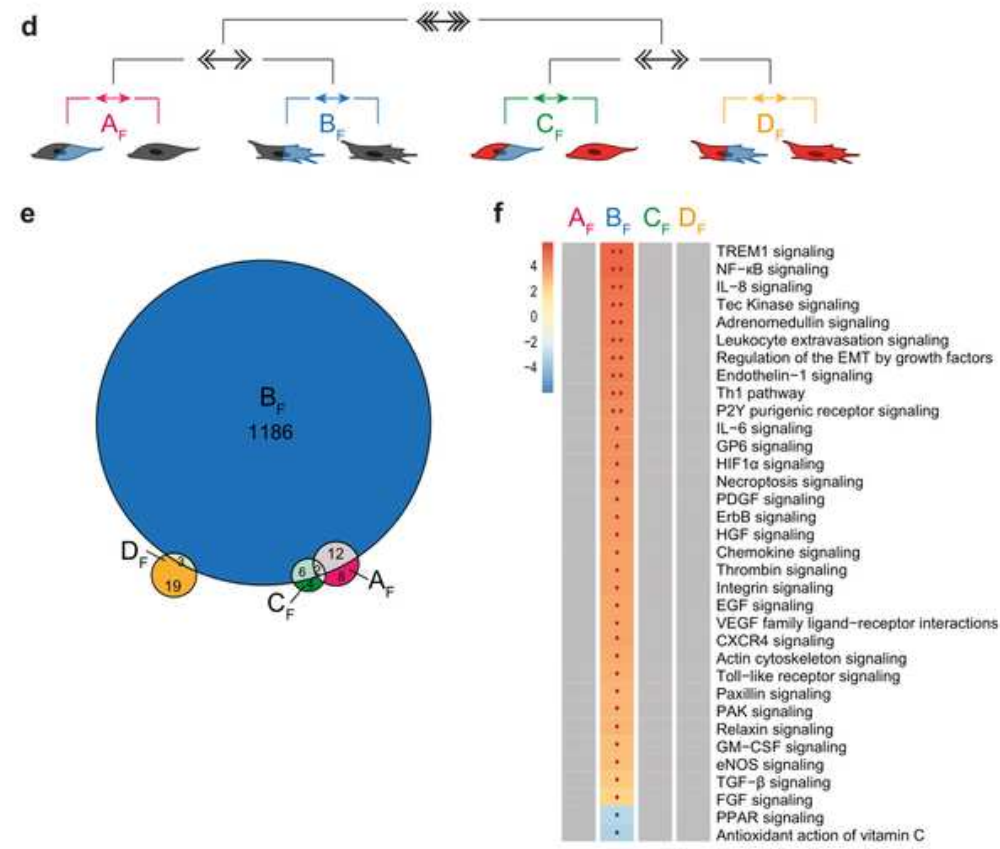

\section{Figure 4}

Role of hypoxia on the global biological response. a, d) Schematic representation of the multi-level approach to study the effect of hypoxia on $\mathrm{M} \varphi$ (panels $\mathrm{a}-\mathrm{c}$ ) and $\mathrm{Fb}$ (panels $\mathrm{d}-\mathrm{f}$ ) in different immune and culture conditions. 1st level compares hypoxic cells to normoxic cells in four different conditions: resting and single culture (comparison $\mathrm{AM} / \mathrm{AF}$ in pink), resting and coculture (BM/BF in blue), LPS+IFNy and single culture (CM/CF in green), LPS+IFNy and coculture (DM/DF in yellow); 2nd level (double arrows) 
compares coculture to single culture conditions (AvsB and CvsD); 3rd level (triple arrows) compares resting to proinflammatory conditions $[(A+B) v s(C+D)]$. b,e) Venn diagrams with $D E G$ s distribution in each 1st level comparison in $M \varphi$ (b) and $F b(e) . c$, f) Pathways enrichment analysis in $M \varphi$ (c) and $F b$ (f) comparisons. Columns represent 1 st level analysis (A-B-C-D), rows report pathways significantly modulated $(\mid z$-score $\mid \geq 2)$ in at least one comparison. Color intensity bar indicates the level of positive (in red) or negative (in blue) enrichment; dots appear only when pathways are significantly enriched ( $z-s c o r e$ $>2 ; \cdot \cdot$ z-score $>4 ; \cdot z$-score $<-2 ; \cdot \cdot z$-score $<-4)$; grey indicates no modulation. See also Fig. S4.
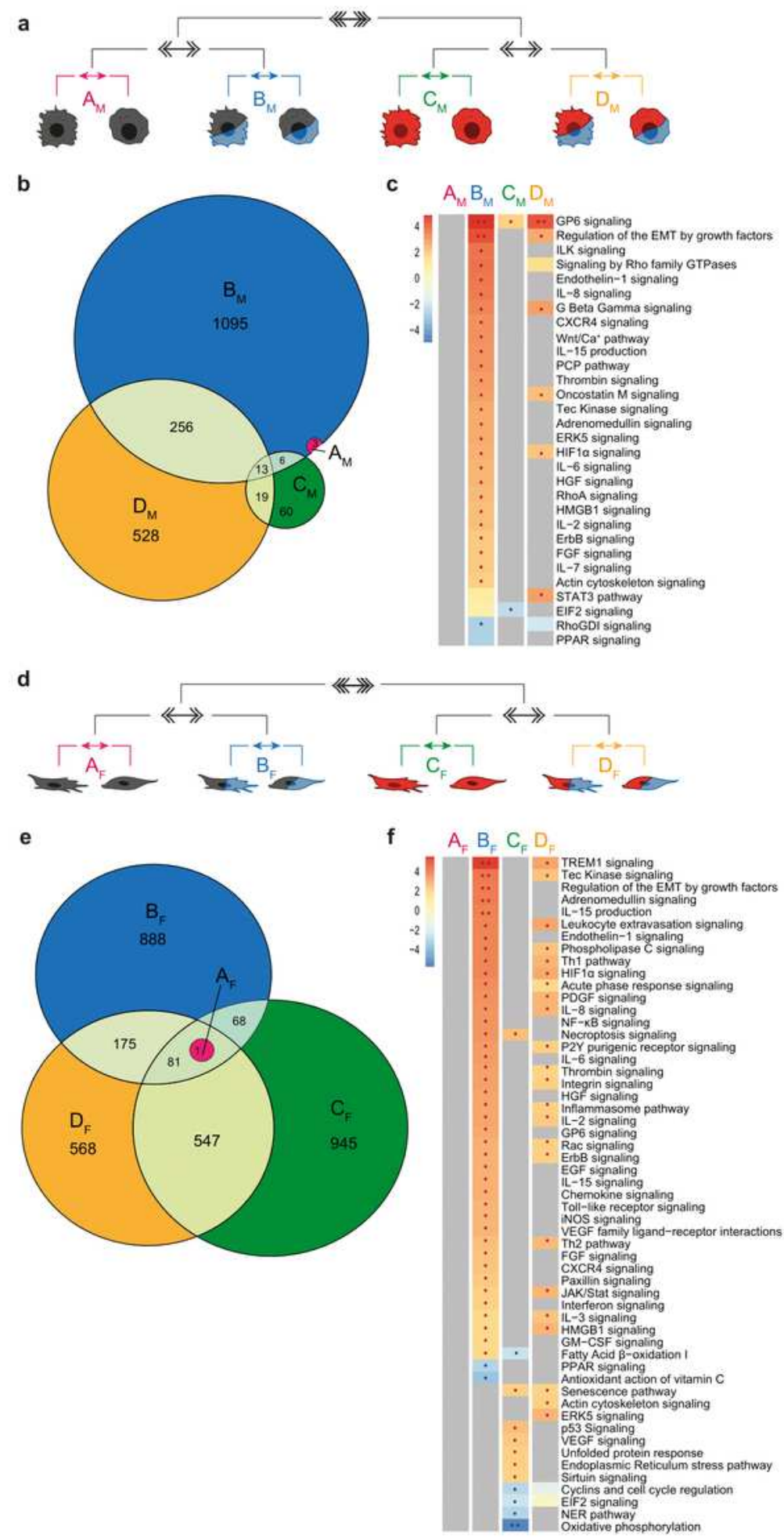


\section{Figure 5}

Role of cellular interplay on the global biological response. a, d) Schematic representation of the multilevel approach to study the effect of $\mathrm{Fb}$ on $\mathrm{M} \varphi$ (panels a-c) and of $M \varphi$ on Fb (panels d-f) in a tissue-like setting with different metabolic and immune conditions. 1 st level compares cocultivated cells to single cultivated cells in four different conditions: in normoxic and resting (comparison AM/AF in pink), in hypoxic and resting (BM/BF in blue), in normoxic and inflamed (CM/CF in green), in hypoxic and inflamed (DM/DF in yellow). 2nd level (double arrows) compares normoxic to hypoxic environments (AvsB and CvsD) and 3rd level (triple arrows) compares resting to proinflammatory conditions $[(A+B) v s(C+D)] . b, e)$ Venn diagrams show the distribution of DEGs in each 1st level comparison in $M \varphi(b)$ and $F b(e) . d, f)$ Pathways enrichment analysis in $M \varphi$ (d) and $F b(f)$ comparisons. Columns represent 1 st level of comparisons (A-B-C-D), rows report pathways significantly modulated (|z-score| $\geq 2$ ) in at least one comparison. Color intensity bar indicates the level of positive (in red) or negative (in blue) enrichment; dots appear only when pathways are significantly enriched $(\cdot z$-score $>2 ; \cdot \cdot z$-score $>4 ; \cdot z$-score $<-2 ; \cdot \cdot z$ score <-4); grey indicates no modulation. See also Fig. S5. 

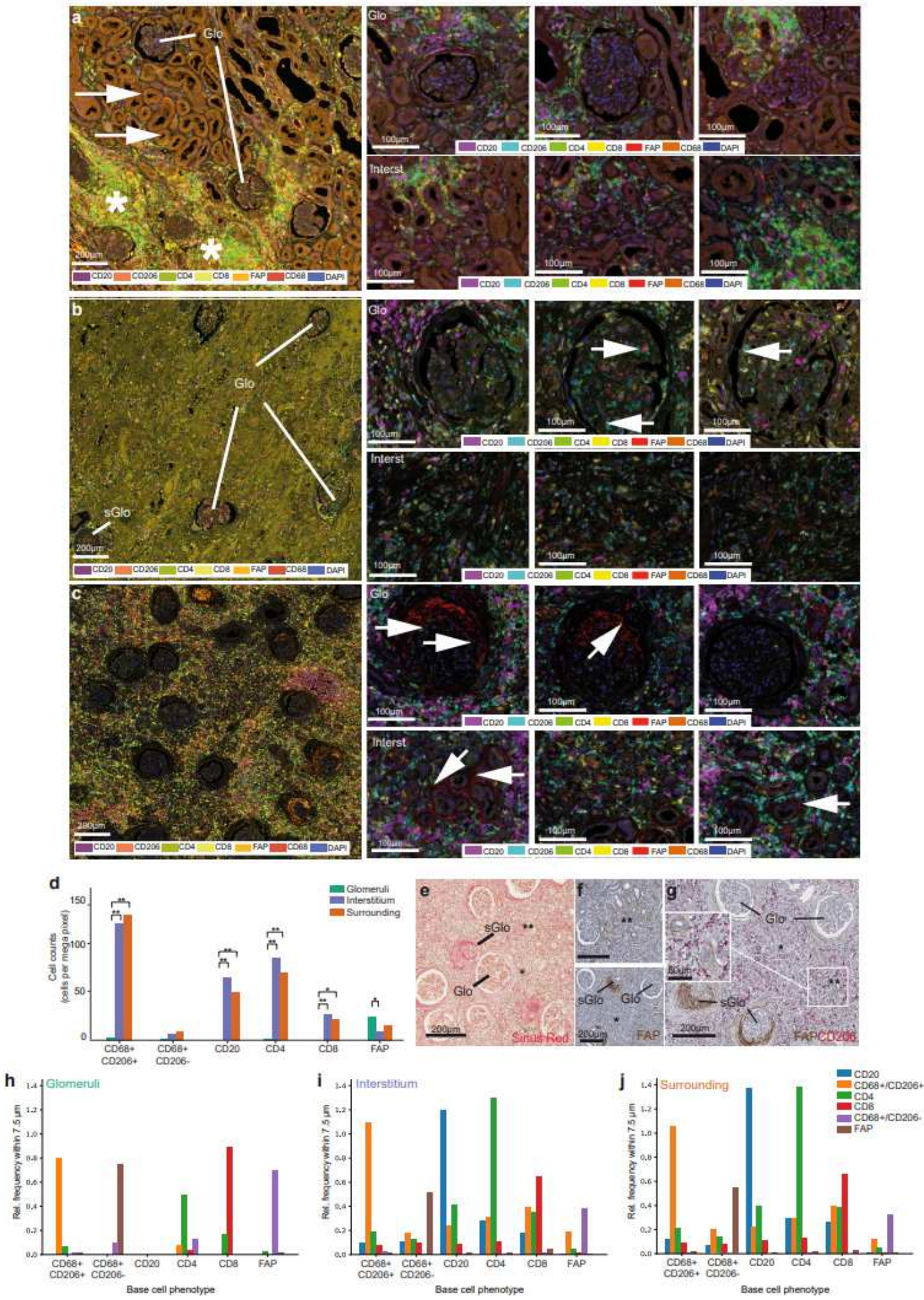

\section{Figure 6}

Different stages of fibrosis and heterogeneity of immune cell infiltrates characterizing distinct anatomical kidney regions. (a-c) Multiplexed immunohistochemistry of transplant nephrectomy samples in (a) acute cell-mediated rejection (TCMR; BANFF type lla), (b) chronic antibody-mediated rejection (ABMR; BANFF type III), and (c) active stage of fibrosis (ca. 90\% IF/TA; BANFF grade III) with signs of chronic rejection that make it difficult to differentiate between TCMR and ABMR. The left panel of (a-c) shows fluorescent 
whole slide scans for overview in tissue context. The right 3 panels show region-specific immune cell phenotyping in multiple fields of view after multispectral unmixing (see color code in right panel of a-c). Representative examples of the glomerular compartment (Glo) and the tubulointerstitial areas (IF/TA) are displayed. Co-existence of almost normal Glo and tubulointerstitial areas (a, left panel, arrows) and severely inflamed regions ( $a$, left panel, asterisks) can be observed. Glomeruli were almost devoid of $M \varphi$ with few exceptions (b, right panel, arrows). In glomeruli, $M \varphi$ were not in direct neighborhood to FAP+ myofibroblasts (Fig. 6c, right panel, arrows). d) Cell density bar plot of different cell types: macrophages (CD68+/CD206+, CD68+/CD206-), T-lymphocytes (CD8+, CD4+), B lymphocytes (CD20+) and fibroblasts $(\mathrm{FAP}+)$, corresponding to $(\mathrm{c})$ in each anatomical compartment (glomeruli = green; interstitium = violet; surrounding $=$ orange; $* p<0.05, * * p<0.01$ ). (e) Sirius red staining (corresponding to $c$ ) showing coexistence of morphologically almost intact Glo and sclerotic glomeruli (sGlo), and tubulointerstitial areas ranging from severe (**) to moderate/low fibrosis $\left({ }^{\star}\right)$. (f) IHC for FAP (corresponding to $\mathrm{c}$ ), showing coexistence of almost normal Glo and areas of massive myofibroblast activation (sGlo), and tubulointerstitial areas ranging from severe $\left({ }^{\star *}\right)$ to moderate/low myofibroblast activation $\left({ }^{\star}\right)$. (g) Duplex immunohistochemistry (corresponding to $c$ ) confirmed the heterogeneous distribution of activated myofibroblasts (FAP+), and alternatively activated CD206+M $\varphi$ in different anatomical regions. (h-j) Neighborhood analysis on multiplexed IHC. For each region, glomeruli (h), interstitium (i) and surrounding (j) is evaluated on the average number of different cell types near $(7.5 \mu \mathrm{m})$ the type of interest (horizontal axis). Under each cell type its absolute number is reported. 
a

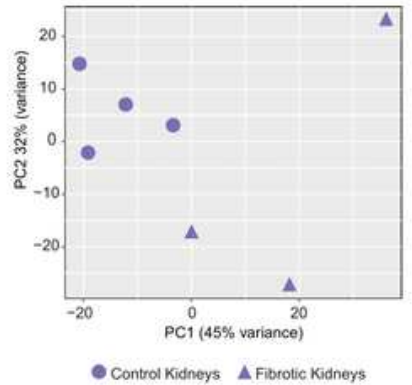

b

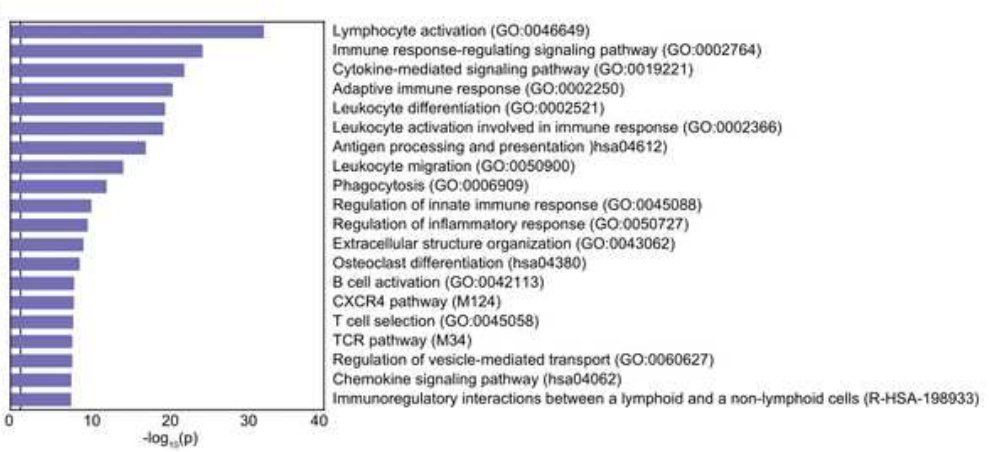

。

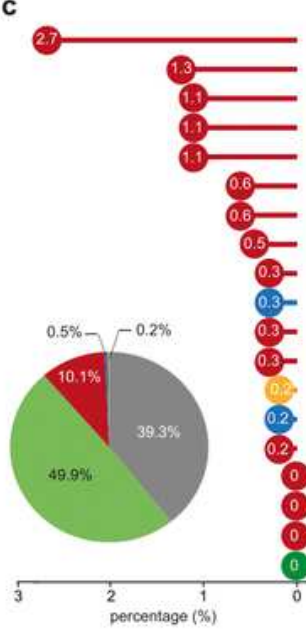

genes associated to inflammation signature

genes associated to $1 \mathrm{~L}-4$ stimulation signature

genes associated to cetl-cell interaction signature

genes associated to hypoxia signature

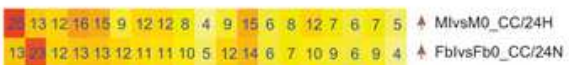

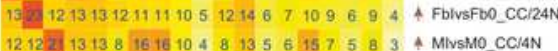
161313.417921211859 .17799117575 A MiveMO CC/24N 1513131721013128491867117584 A MivsMo_SC/24H 9128910149782121046877882 A FblvsFbo_SC/24H $12.11161213920149 .4 \quad 81345158$ 6 833 4 MlvsMO_CC/4H 1211181112714199371245157583 A MlvsMO_SC/4N 810108889913388845896102 4 FblvsFbo_CC/4N

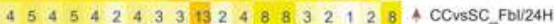

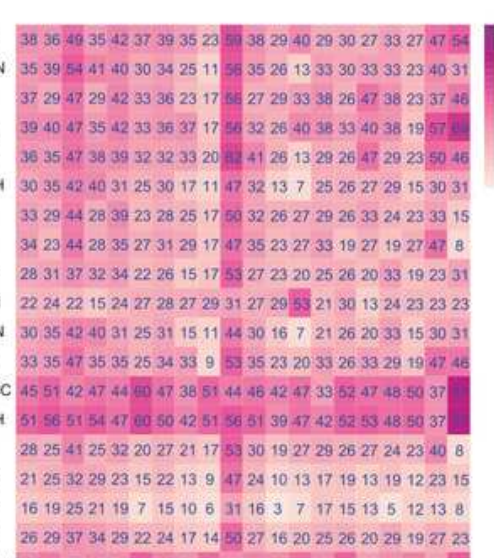

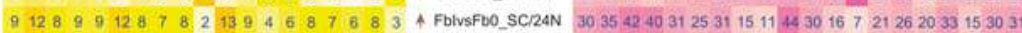

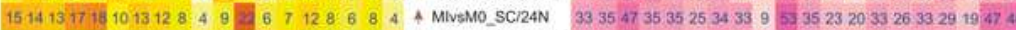

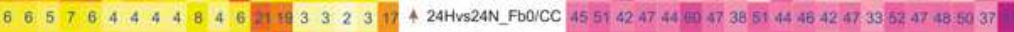

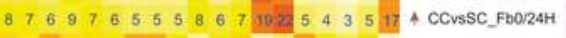
12101511118151583381235167572 A MlvsMO SC $/ 4 \mathrm{H}$

797777879278347106824 FblvsFbo_CC/4H 7987888810288835786112 A FblvsFbo SC/4N $262937342922241714 \quad 5227162025262029192723$ $\begin{array}{lllllllllllllllllll}5 & 4 & 3 & 5 & 4 & 2 & 3 & 3 & 2 & 8 & 3 & 4 & 17 & 17 & 2 & 2 & 1 & 2 & 18\end{array}$ + FbFusFbo_CC/24H

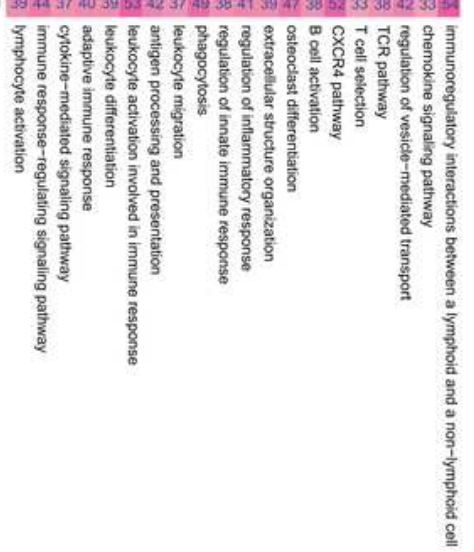

\section{Figure 7}

Gene overlap analysis of ex vivo tubulointerstitial area from fibrotic kidneys with in vitro signatures. a) PCA of ex vivo interstitium samples. Triangles indicate fibrotic kidneys, dot control kidneys. b) Bar graph reports pathways significantly enriched in fibrotic vs control interstitium samples as calculated by Metascape software. c) Overlap analysis was performed comparing DEGs of ex vivo fibrotic vs control interstitium with DEGs of each in vitro comparison (which defined 78 corresponding signatures). Pie chart 
reveals that $49.9 \%$ of ex vivo signature is explained by at least one in vitro signature (in light green); $10.8 \%$ is specifically explained by only one in vitro signature and different colors are referred to the discriminating variable: inflammation in red, IL-4 stimulation in green, cell-cell interaction in blue, hypoxia in yellow. The remaining $39.3 \%$ of the ex vivo interstitium signature (633 genes) is not explained by in vitro model (in grey). Dot chart reports on the vertical axis 19 in vitro signatures enriched in ex vivo model and their relative contribution with unique expressed genes: white numbers in each dot represent relative percentages on the totality of explained and not explained genes. The central heatmap highlights the overlapping percentage of the same 19 in vitro signatures on the ex vivo signature. On the diagonal, numbers indicate the overlapping percentage of each signature; the other numbers explain the overlapping percentage shared between two in vitro and the ex vivo signatures. Color intensity indicates the overlapping percentage level referred to ex vivo signature. Arrows near each in vitro signature indicate the positive (red) or negative (blue) enrichment of the first term of in vitro comparison (see also Fig. S7). The heatmap on the right reports Metascape pathways in ex vivo comparison (panel b) with the percentage of enrichment given by each in vitro signature (on the left). 
a

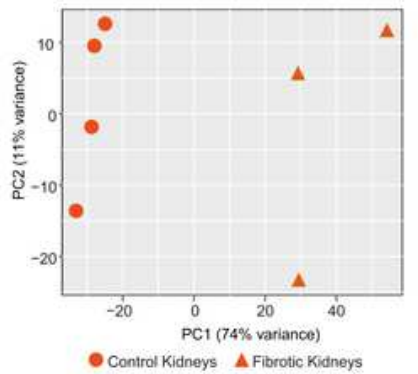

c

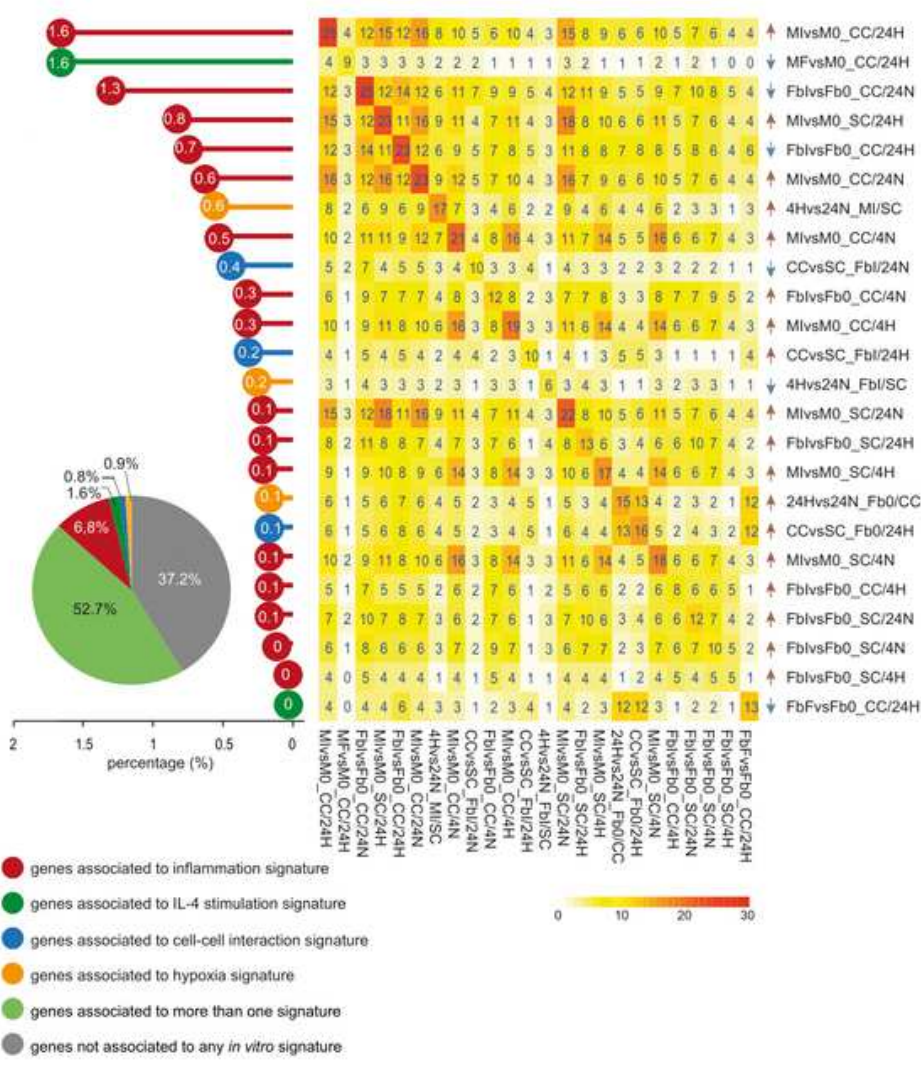

b

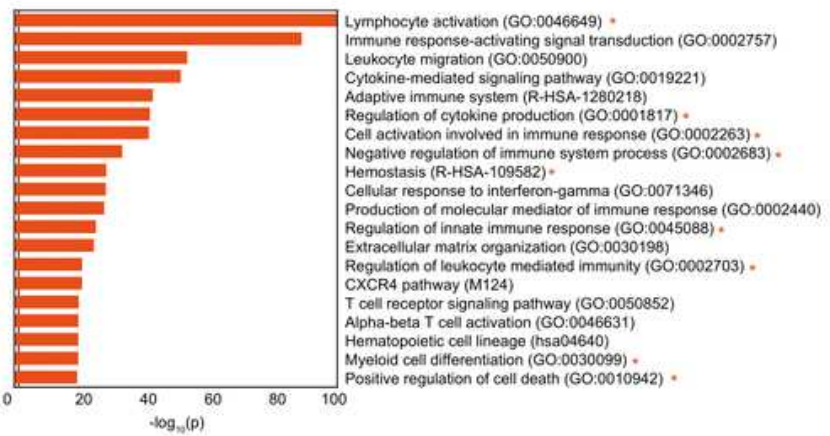

$30 \quad 33 \quad 33$ 45 $31 \quad 3936 \quad 3934 \quad 552147 \quad 35 \quad 43 \quad 3134 \quad 3541 \quad 2637$

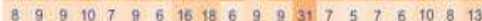

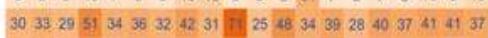
$3135 \quad 32 \quad 4730 \quad 36 \quad 37 \quad 36 \quad 33 \quad 56 \quad 2850 \quad 34 \quad 45 \quad 29 \quad 37.3736 \quad 3140$

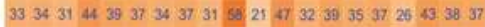
$30 \quad 33 \quad 31 \quad 43 \quad 36 \quad 35 \quad 35 \quad 41 \quad 28 \quad 5321 \quad 49 \quad 36 \quad 42$ 31 39 40 37 29 a5 $181917 \quad 1722 \quad 25232221 \quad 1722 \quad 2623271826192214 \quad 22$

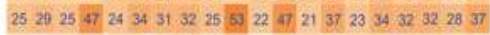
$\begin{array}{llllllllllllllllllll}8 & 8 & 8 & 14 & 9 & 8 & 10 & 11 & 8 & 19 & 3 & 14 & 11 & 10 & 13 & 14 & 13 & 13 & 9 & 10\end{array}$ $17 \quad 22 \quad 15 \quad 38 \quad 18 \quad 25 \quad 21 \quad 27 \quad 16 \quad 5720 \quad 36 \quad 2129 \quad 13 \quad 27 \quad 24 \quad 23 \quad 21 \quad 29$ $242820 \quad 42 \quad 2033 \quad 2432 \quad 25 \quad 49 \quad 18 \quad 42 \quad 19351932 \quad 3426 \quad 27 \quad 35$ $\begin{array}{llllllllllllllllllll}18 & 17 & 17 & 19 & 21 & 20 & 21 & 17 & 19 & 12 & 13 & 21 & 18 & 28 & 24 & 23 & 28 & 22 & 24 & 17\end{array}$

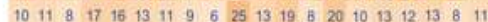

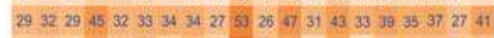
$1924194124 \quad 28 \quad 202917 \quad 0028 \quad 40 \quad 15 \quad 37 \quad 12 \quad 28 \quad 28 \quad 28 \quad 21 \quad 26$ $20 \quad 27 \quad 24 \quad 45 \quad 1933 \quad 23.29 \quad 2152.18 \quad 46 \quad 1830 \quad 24 \cdot 31 \quad 28 \quad 28 \quad 27 \quad 34$ 33 31 $31 \quad 32 \quad 4137 \quad 46 \quad 35 \quad 2839 \quad 2939$ 18 $51.41284134 \quad 44 \quad 27$ $343431 \quad 3844 \quad 40 \quad 46 \quad 3731.4930 \quad 47 \quad 16 \quad 55 \quad 3936 \quad 43 \cdot 37 \quad 42 \quad 30$ $24 \quad 28 \quad 264518 \quad 3328292748 \quad 2044 \quad 1936 \quad 2228292926 \quad 37$ $12 \quad 1713 \quad 311420142174512311023 \quad 1023 \quad 16 \quad 19 \quad 13 \quad 21$ $1824194025 \quad 27.2129 .16 \quad 0126 \quad 381636 \quad 12 \quad 282528 \quad 1924$ $16 \quad 21.15 \quad 38 \quad 18 \quad 24 \quad 1924 \quad 1452 \quad 21 \quad 36 \quad 17 \quad 30 \quad 13 \quad 28 \quad 22 \quad 23 \quad 19 \quad 25$

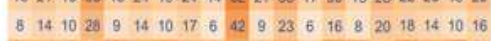

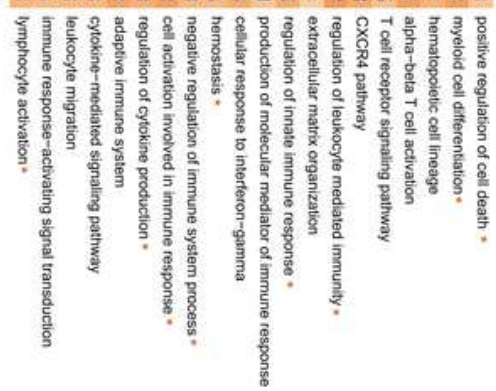

\section{Figure 8}

Gene overlap analysis of ex vivo glomeruli surrounding area from fibrotic kidneys with in vitro signatures. a) PCA of ex vivo glomeruli surrounding area samples. Triangles indicate fibrotic kidneys, dot control samples. b) Bar graph reports pathways significantly enriched in pathological vs control surrounding samples as calculated by Metascape software. c) Overlap analysis was performed comparing DEGs of ex vivo fibrotic vs control surrounding areas with DEGs of each in vitro comparison (which defined 78 
corresponding signatures). Pie chart reveals that $52.7 \%$ of ex vivo signature is explained by at least one in vitro signature (in light green); $10.1 \%$ is specifically explained by only one in vitro signature and different colors are referred to the discriminating variable of in vitro signatures: inflammation in red, IL-4 stimulation in green, cell-cell interaction in blue, hypoxia in yellow. The remaining $37.2 \%$ of the surrounding ex vivo signature (2,685 genes) is not explained by in vitro model (in grey). Dot chart reports on the vertical axis 24 in vitro signatures enriched in ex vivo model and their relative contribution with unique expressed genes: white numbers in each dot represent relative percentages on the totality of explained and not explained genes. The central heatmap highlights the overlapping percentage of the same 24 in vitro signatures. On the diagonal, numbers indicate the overlapping percentage of each signature; the other numbers explain the overlapping percentage shared between two in vitro and the ex vivo signatures. Color intensity indicates the overlapping percentage level referred to ex vivo signature. Arrows near each in vitro signature indicate the positive (red) or negative (blue) enrichment of the first term of in vitro comparison (see also Fig. S7). The heatmap on the right reports Metascape pathways in ex vivo comparison (panel $b$ ) with the percentage of enrichment given by each in vitro signature (on the left). Orange stars highlight pathways that are specifically enriched in surrounding nephrectomies and are not shared with other renal districts (interstitium, glomeruli). 
a

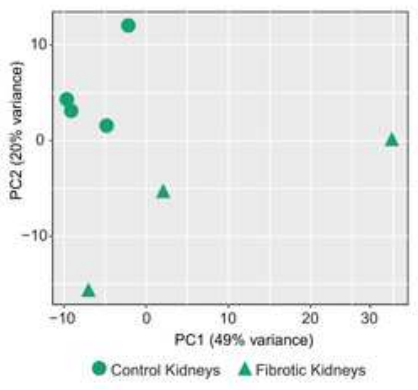

b

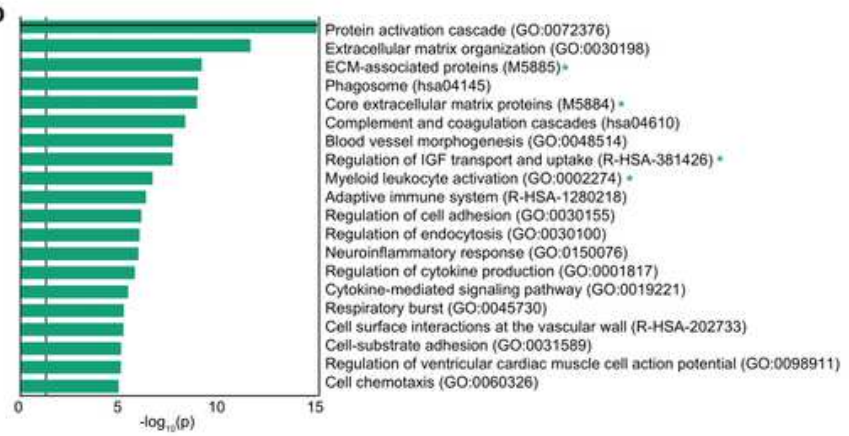

c

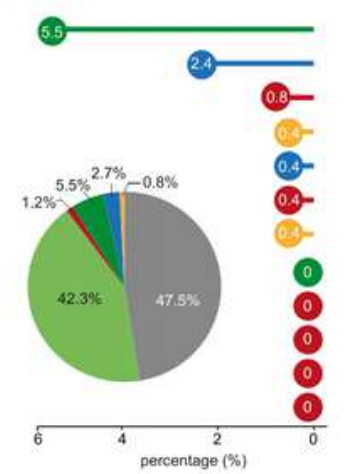

- genes associated to inflammation signature

genes associated to IL-4 stimulation signature

genes associated to cell-cell interaction signature

genes associated to hypoxia signature

18 $\begin{array}{llllllllllll}2 & 3 & 2 & 2 & 2 & 5 & 1 & 9 & 3 & 4 & 4 & 4\end{array}$ MFvsmo_CC $24 \mathrm{H}$

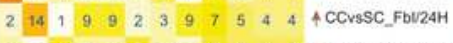
$\begin{array}{lllllllllllllll}3 & 1 & 9 & 3 & 3 & 6 & 3 & 2 & 6 & 5 & 5 & 5 & 4 & \text { FblvsFBO_SC/24H }\end{array}$

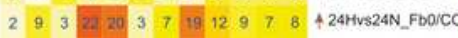

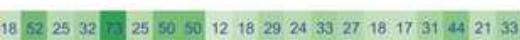

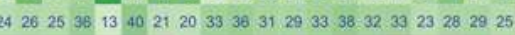

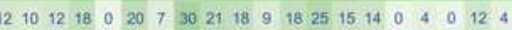

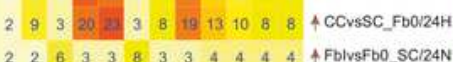

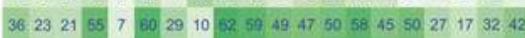

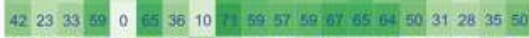
$\begin{array}{lllllllllllllllllllllllllllllllll}2 & 2 & 6 & 3 & 3 & 8 & 3 & 3 & 4 & 4 & 4 & 4 & \text { A F FlvsFbo_SC/24N } 11 & 10 & 8 & 18 & 0 & 25 & 14 & 30 & 21 & 14 & 6 & 18 & 17 & 15 & 14 & 0 & 4 & 0 & 15 & 8\end{array}$

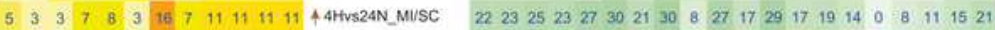

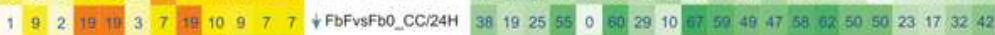

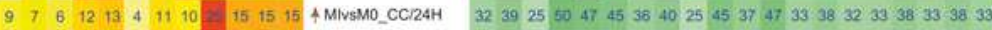

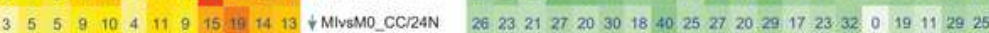

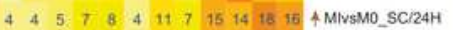

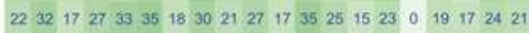

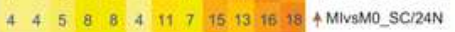

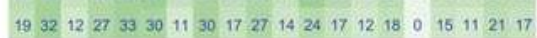
mpengengen mong

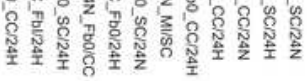
IIIIIIIIIIIII

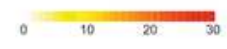

genes not associated to any in vitro signature

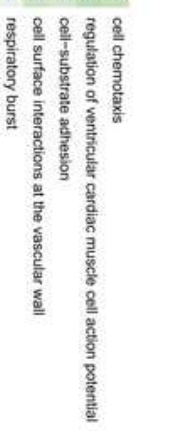

\section{Figure 9}

Gene overlap analysis of ex vivo glomeruli from fibrotic kidneys with in vitro signatures. a) PCA of ex vivo glomeruli samples. Triangles indicate fibrotic kidneys, dot control samples. b) Bar graph reports pathways significantly enriched in fibrotic vs control glomeruli samples as calculated by Metascape software. c) Overlap analysis was performed comparing DEGs of ex vivo fibrotic vs control glomeruli with DEGs of each in vitro comparison (which defined 78 corresponding signatures). Pie chart reveals that 
$42.3 \%$ of ex vivo signature is explained by at least one in vitro signature (in light green); $10.2 \%$ is specifically explained by only one in vitro signature and different colors are referred to the discriminating variable of in vitro signatures: inflammation in red, IL-4 stimulation in green, cell-cell interaction in blue, hypoxia in yellow. The remaining $47.5 \%$ of the ex vivo glomeruli signature ( 255 genes) is not explained by in vitro model (in grey). Dot chart reports on the vertical axis 12 in vitro signatures enriched in ex vivo model and their relative contribution with unique expressed genes: white numbers in each dot represent relative percentages on the totality of explained and not explained genes. The central heatmap highlights the overlapping percentage of the same 12 in vitro signatures on the ex vivo signature. On the diagonal, numbers indicate the overlapping percentage of each signature; the other numbers explain the overlapping percentage shared between two in vitro and the ex vivo signatures. Color intensity indicates the overlapping percentage level referred to ex vivo signature. Arrows near each in vitro signature indicate the positive (red) or negative (blue) enrichment of the first term of in vitro comparison. See also Fig. S7. The heatmap on the right reports Metascape pathways in ex vivo comparison (panel b) with the percentage of enrichment given by each in vitro signature (on the left). Green stars highlight pathways that are specifically enriched in glomeruli nephrectomies and are not shared with other renal districts (interstitium, surrounding area). 

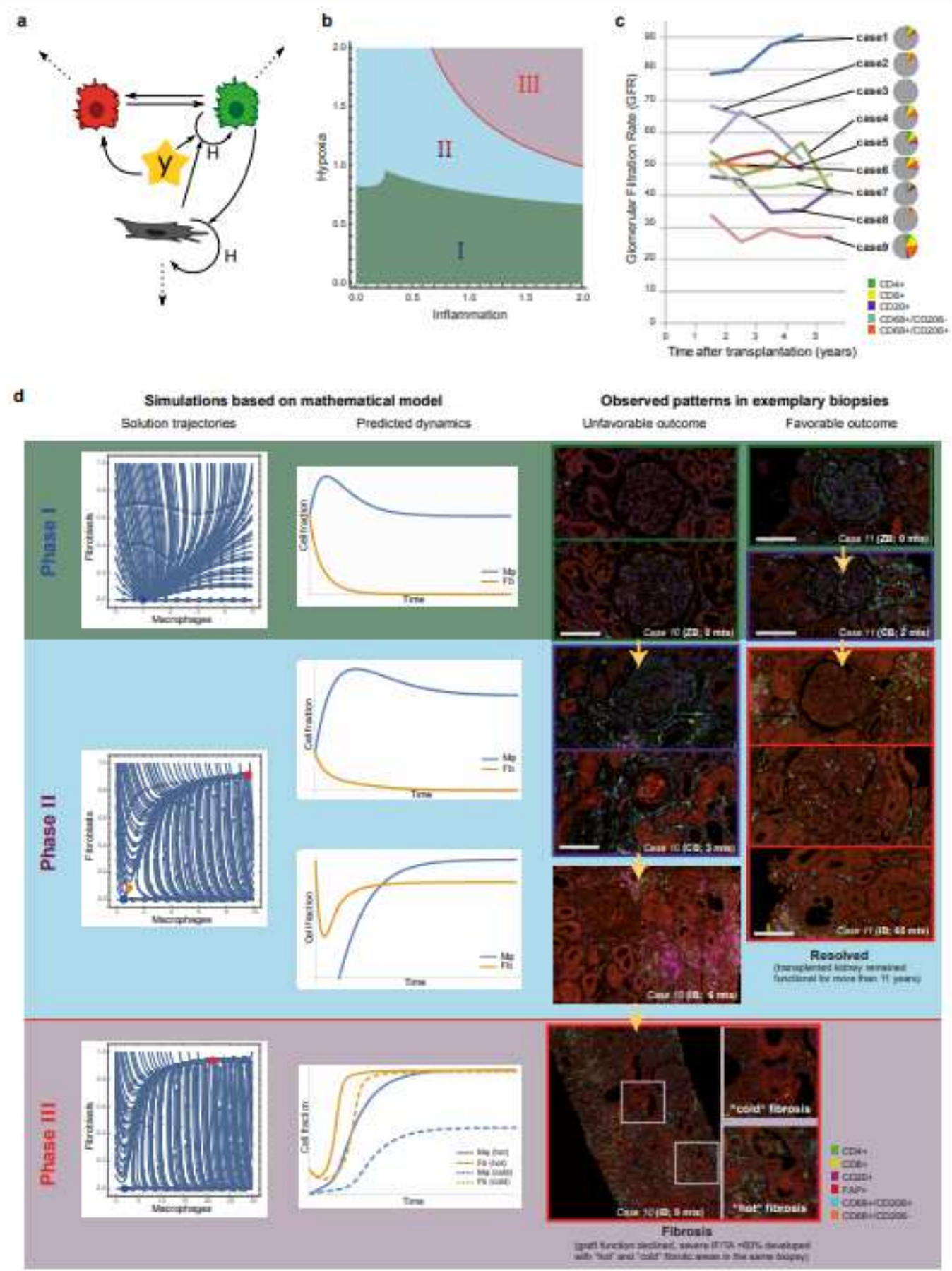

\section{Figure 10}

Mathematical model based on in vitro data and exemplary clinical observations reflecting the predicted outcomes. a) Schematic representation of $M \varphi$ (MI in red and MF in green) and Fb interactions under hypoxia $(\mathrm{H})$ and inflammation $(\mathrm{Y})$. b) Phase diagram of mathematical prediction. Three phases (I-II-II) were identified on different levels of inflammation (horizontal axis) and hypoxia (vertical axis). The red line between phases II and III is the separatrix. c) Nine exemplary cases (case 1-9) reflecting the stable "Phase I": The renal function, represented by the glomerular filtration rate (GFR) over time after transplantation, can stay stable over many years, despite substantial infiltration by immune cells, 
including many macrophages, in a protocol biopsy taken around the time point $\sim 6$ months after transplantation. Pie charts describe the individual immune cell infiltrate (lymphocytes and $M \varphi$ ) evaluated by quantitative digital pathology after multiplex immunohistochemistry. The percentage of immune cells (color code in the figure legend indicates the immunophenotype) in comparison to all nucleated cells in the biopsy as defined by DAPI nuclear staining (grey area in the pie charts) can massively vary between almost no inflammation (e.g., case 3, 8) and abundant infiltrates (e.g., cases 5, 6, 9). Nevertheless, the renal function stays stable over 2-5 years, indicating that the inflammatory process resolved to a nonfibrotic state in sufficiently large areas of the transplanted kidney. d) Graphical depiction of in-silico simulations (left panels) show the solution trajectory and the predicted dynamics of $\mathrm{M} \varphi$ and $\mathrm{Fb}$ depending on time (horizontal axis) and cell fraction (vertical axis) for each of the three phases as predicted by the mathematical model in more detail. The right panel shows corresponding representative clinical constellations that confirmed the hypotheses generated by computer simulations, based on the exemplary cases 10 (unfavorable outcome with irreversible "hot" and "cold" fibrosis) and case 11 (favorable outcome despite "borderline" immune infiltration). The color legend indicates the different cell types as detected my multiplexed immunohistochemistry. Note the abundant CD206+ macrophages in the indicated biopsy of case 11 in "Phase II" in the surrounding area (upper image), inside the glomerulus (middle image) and in the interstitial compartment (bottom image), and in case 10 in "Phase III" the coexistence of areas with many CD206+ macrophages ("hot" fibrosis) and low density of this phenotype ("cold" fibrosis) co-localized with myofibroblast activation (FAP+ cells) in the same biopsy as predicted by in-silico modeling.

\section{Supplementary Files}

This is a list of supplementary files associated with this preprint. Click to download.

- Supplementaryinformation.pdf 